\title{
EL EFECTO DEL FUEGO Y LAS CARACTERÍSTICAS TOPOGRÁFICAS SOBRE LA VEGETACIÓN Y LAS PROPIEDADES DEL SUELO EN LA ZONA DE TRANSICIÓN ENTRE BOSQUES Y PASTIZALES DE LAS SIERRAS DE Córdoba, Argentina
}

\author{
MELISA A. GIORGIS ${ }^{1,2}$, ANA M. CINGOLANI ${ }^{1}$ y MARCELO CABIDO' ${ }^{1}$
}

\begin{abstract}
Summary: The effect of fire and topographic features on vegetation and soil properties in woodlands and grasslands transition in Córdoba mountains, Argentina. Under the currently context of climate change the dynamic of the transition zone between forests and grasslands has a fundamental role at both scientific level and in planning and ecosystem management. In this study, we evaluated the combined effect of fire, slope aspect and topographic position on vegetation and soil characteristics of the Córdoba Mountains in the transition zone between woodlands and grasslands. We selected four areas with different post-fire age. In each area we selected all possible combinations of slope aspects and topographic positions. In each site we performed floristic surveys and analyzed a composite sample of soil. Fire was the main factor associated with the different characteristics analyzed (physiognomy, floristic composition, soil characteristics), and separated burned from unburned sites. Slope aspect also contributed, conditioning physiognomy, floristic composition and soil characteristics. However we did not found a straight relationship between the recovery of tree cover and the time after the last fire, indicating that other factors such as geomorphology, land use history, and herbivory would also condition post-fire recovery of these systems.
\end{abstract}

Key words: Ecotone, floristic composition, mountain woodland, mountains, physiognomy, treeline.

Resumen: En el contexto actual de cambio climático, el estudio de la dinámica de las zonas de transición entre bosques y pastizales tiene un papel fundamental, tanto a nivel científico como en la planificación del manejo de los ecosistemas. En este trabajo evaluamos el efecto combinado del fuego, la exposición de ladera y la posición topográfica sobre la vegetación y los suelos en una zona de transición entre bosques y pastizales en las montañas de Córdoba. Para ello elegimos cuatro áreas con diferentes edades post-fuego. En cada sector seleccionamos todas las combinaciones posibles de exposición y posición topográfica. En cada sitio realizamos un relevamiento florístico completo y analizamos una muestra compuesta de suelo. El tiempo transcurrido después del último incendio fue el principal factor determinante de las distintas características analizadas (fisonomía, composición florística, riqueza y propiedades del suelo), diferenciando sitios quemados recientemente de los no quemados. La exposición de ladera también tuvo un rol importante condicionando la fisonomía, la composición florística y las propiedades de los suelos. Sin embargo, no encontramos una relación directa entre la recuperación de la cobertura arbórea y el tiempo transcurrido después del último incendio, indicando que otros factores como las características geomorfológicas, historia de uso y herbivoría, estarian condicionando la recuperación post-fuego de estos sistemas.

Palabras clave: bosque serrano, composición florística, ecotono, fisonomía, límite de bosques, montañas.

1. Instituto Multidisciplinario de Biología Vegetal, CONICET- FCEFyN, Universidad Nacional de Córdoba, Córdoba, Argentina.

2 e-mail: melisagiorgis@hotmail.com 


\section{INTRODUCCIÓN}

La dinámica de las zonas de transición entre ecosistemas de bosques y pastizales se ha transformado en un foco de especial atención frente a escenarios actuales de cambio climático (Dullinger et al., 2004; Batllori \& Gutiérrez, 2008; Harsch et al., 2009). Tradicionalmente las características climáticas, tanto a escala global como local, eran consideradas los principales factores determinantes de esta dinámica (Swanson et al., 1988; Reinhardt et al., 2010). Sin embargo, el fuego es uno de los factores más importantes que afecta la dinámica de la vegetación (Bond \& Keeley, 2005) y una de las principales herramientas utilizadas por el hombre para transformar comunidades boscosas en sistemas abiertos aptos para actividades agrícolas y ganaderas (Reich et al., 2001; Grau, 2003; Bowman et al., 2009). Este disturbio es en la actualidad, e incluso durante los últimos miles de años, uno de los principales factores moduladores del paisaje en numerosas zonas de transición entre bosques y pastizales (Grau \& Aragon, 2000; Villalba et al., 2005; Martin et al., 2007; Sherman et al., 2008; Genries et al., 2009; Coop et al., 2010).

El fuego produce una remoción directa de la biomasa vegetal condicionando la supervivencia de las plantas, su crecimiento posterior y el establecimiento de nuevos individuos. De este modo, produce grandes modificaciones en la fisonomía (Moreira, 2000; Eugene \& Lloret 2004; Fulé \& Laughlin 2007), la composición florística (ViláCabrera et al., 2008; Coop et al., 2010) y la riqueza de especies (DeGrandpré et al., 1993; Rees \& Juday, 2002). Es ampliamente reconocido que las formas de vida más negativamente afectadas por el fuego son los árboles y enredaderas, que pueden tardar mucho tiempo en volver a establecerse y recuperar su biomasa anterior al fuego. Por el contrario, las gramíneas y las hierbas anuales suelen responder de forma positiva, alcanzando poco tiempo después del fuego abundancias mayores a las que tenían con anterioridad (Eugene \& Lloret, 2004). Además, debido a la reducción de la cobertura arbórea y a la mayor disponibilidad de luz, la riqueza de especies generalmente aumenta poco tiempo después del fuego (DeGrandpré et al., 1993; Rees \& Juday, 2002; Coop et al., 2010). Es decir, que si no hay limitaciones importantes en la dispersión de propágulos, y si después del fuego ningún otro disturbio produce disrupciones importantes en la dinámica sucesional del sistema, las distintas características de la vegetación estarán directamente relacionadas con el tiempo transcurrido después del incendio (Gibson \& Hulbert, 1987; Lecomte et al., 2006).

El fuego no sólo afecta a la vegetación, sino también a las propiedades del suelo (Kunst \& Bravo, 2003; Albanesi \& Anriquez, 2003; Eugene \& Lloret, 2004). El efecto más evidente se manifiesta sobre la fertilidad edáfica, al producirse una violenta mineralización de la materia orgánica. Además, la actividad microbiana, y consecuentemente el ciclado de nutrientes, se ven drásticamente modificados (Gonzalez et al., 1999; Abril \& Gonzalez, 1999; Albanesi \& Anriquez, 2003). Numerosos factores pueden condicionar el efecto inmediato del fuego y la recuperación de las propiedades del suelo después de un incendio (Belnap \& Eldridge, 2001). Entre estos factores, las características de la vegetación es uno de los más importantes. Por un lado, la estructura de la vegetación preexistente condiciona la intensidad de los incendios, y por otro, la identidad y características de las especies condicionan la capacidad de recuperación del sistema y por ende la dinámica sucesional que se produce después del disturbio (Johansen, 2003). Por ello, a medida que transcurre el tiempo después de un incendio, y simultáneamente con el avance de la sucesión vegetal, se podría esperar una paulatina recuperación del suelo (Cerda \& Doerr, 2005).

En terrenos montañosos la recuperación postfuego está influenciada por las características geomorfológicas del terreno, como la exposición de ladera y la posición topográfica (Gibson \& Hulbert, 1987; Swanson et al., 1988; Turner \& Romme, 1994; Kramer et al., 2001; Kulakowski \& Veblen, 2002; Collins et al., 2007; Sherman et al., 2008). Estas variables condicionan las características ambientales del sitio, como la temperatura y la humedad, afectando los patrones de germinación y la tasa de crecimiento de las distintas especies vegetales, así como las interacciones entre las mismas. Por ende, las características del sitio pueden influir sobre la fisonomía, composición florística, riqueza de especies y propiedades del suelo en las diferentes etapas sucesionales (Lavee et al., 1995; Rolling et al., 2002).

La vegetación de las montañas del Centro de Argentina pertenece al Distrito Chaqueño Serrano 
(Cabrera, 1979). En las montañas de Córdoba, a medida que se asciende en altitud, se observan cambios en la fisonomía, composición florística y riqueza de especies (Cabido et al., 1991; Funes \& Cabido, 1995; Giorgis et al., 2011a). Varios trabajos han destacado la ocurrencia de pisos de vegetación a lo largo de este gradiente altitudinal (Kurtz, 1904; Sayago, 1969; Luti et al., 1979). De ellos, el más difundido es el de Luti et al., (1979), en donde se diferencian tres pisos: el Bosque Serrano en la parte más baja, seguido por el Matorral Serrano, también llamado Arbustal de Altura o "Romerillal", y finalmente el piso de los Pastizales y Bosquecillos de Altura, en el sector más alto de las sierras. En el Bosque Serrano de Córdoba, las especies arbóreas dominantes en los estadios sucesionales más avanzados son Lithraea molleoides (Vell.) Engl., Zanthoxylum coco Gillies ex Hook. f. et Arn., y en algunos sectores Schinopsis marginata Engl. (Luti et al., 1979; Giorgis et al., 2011a). En el Romerillal, la especie dominante es un arbusto, Heterothalamus alienus (Spreng.) Kuntze, conocido vulgarmente como romerillo (Luti et al., 1979). Recientemente se ha sugerido que a escala regional, el Romerillal no es un piso característico y continuo de las montañas de Córdoba, siendo en muchos sectores el pastizal la fisonomía en contacto con el sector superior del Bosque Serrano (Giorgis et al., 2011a). En estas montañas el fuego es un fenómeno recurrente en el paisaje (Miglietta, 1994; Renison et al., 2002; Gurvich et al., 2005, Giorgis et al., 2011a). No obstante, las descripciones clásicas de la vegetación serrana atribuyen el límite de distribución del bosque a factores mesoclimáticos asociados a la topografía (altitud, exposición, posición topográfica, Kurtz, 1904; Seckt, 1940; Sayago, 1969; Montaña, 1975; Luti et al., 1979; Montaña \& Ezcurra, 1982). A pesar de la alta recurrencia de incendios, en el centro de Argentina existen pocos estudios que exploren el efecto del fuego sobre la distribución del Bosque Serrano. Si bien algunos autores han abordado la temática en relación a la vegetación (Miglietta, 1994; Gurvich et al., 2005; Verzino et al., 2005, Mari, 2006; Torres, 2012) y al suelo (Abril \& Gonzalez, 1999), ninguno de ellos ha evaluado los efectos del fuego en la franja altitudinal de transición entre el bosque y el pastizal.

Evidencias recientes sugieren que en la actualidad no hay limitaciones climáticas al establecimiento de especies leñosas del bosque a lo largo de todo el gradiente altitudinal de las Sierras de Córdoba. Por un lado, el límite de crecimiento de los árboles a escala global ocurre cuando la temperatura media durante la estación de crecimiento es inferior a $6{ }^{\circ} \mathrm{C}$ (Körner \& Paulsen, 2004), mientras que en Córdoba la temperatura media anual es de 7,4 ${ }^{\circ} \mathrm{C}$ en la parte más alta de las Sierras $(2700 \mathrm{~m}$ s.n.m., Marcora et al., 2008). Es decir que el límite fisiológico encontrado a nivel mundial es claramente superado a nivel local. Por otro lado, evidencias experimentales sugieren que no hay limitaciones al establecimiento y germinación de especies leñosas nativas a lo largo de todo el gradiente altitudinal (Pais Bosch et al., 2012; Marcora et al., 2013). En este contexto, planteamos como hipótesis que las características de la vegetación y el suelo en la zona de transición bosque-pastizal están más fuertemente relacionadas con el tiempo transcurrido después del último fuego, que con factores topográficos como la exposición de la ladera y la posición topográfica. Para poner a prueba esta hipótesis, nos propusimos como objetivo comparar las características de la vegetación (fisonomía, composición florística, riqueza) y las propiedades del suelo entre sitios con distintas combinaciones de edades post-fuego (es decir tiempo transcurrido después del último fuego), exposición de ladera y posición topográfica en una zona de transición entre el Bosque Serrano y los Pastizales de Altura de las Sierras de Córdoba.

Sobre la base de los antecedentes expuestos, formulamos las siguientes predicciones: (1) sitios con diferentes edades post-fuego presentarán mayores diferencias en las características de la vegetación y propiedades del suelo que sitios con distinta topografía pero similar edad post-fuego; (2) sitios con mayor edad post-fuego tendrán una mayor cobertura del estrato arbóreo y una menor cobertura del estrato herbáceo que sitios con menor edad post-fuego, y (3) sitios con edades intermedias post-fuego tendrán una mayor riqueza de especies que sitios sin quemar y sitios con muy cortas edades post-fuego.

\section{Métodos}

Área de estudio

Las Sierras Pampeanas de Córdoba están integradas principalmente por un basamento plutónico metamórfico compuesto por metamorfitas 
de grano grueso (gneises y migmatitas) (Gordillo, 1979), intruidas en el Paleozoico por batolitos graníticos (Rapela, 1982). Estas montañas pueden dividirse en cuatro grandes unidades: Sierras del Norte, Sierras Chicas-Las Peñas, Sierras Grandes-Comechingones, y las Sierras de PochoGuasapampa, que se extienden desde los $29^{\circ} 00^{\prime}$ $\mathrm{S}$ hasta los $30^{\circ} 30^{\prime} \mathrm{S}$, comprendiendo de Norte a Sur $430 \mathrm{~km}$ y $110 \mathrm{~km}$ en sentido Este-Oeste (Capitanelli, 1979; Carignano, 1999). Su altitud varía aproximadamente entre 400 y 2790 m s.n.m. en el "Cerro Champaquí", máxima elevación de las sierras.

Puntualmente, el estudio se realizó en la ladera oriental de las Sierras Chicas-Las Peñas (Lat $31^{\circ} 07^{\prime} \mathrm{S}$ y Long $\left.64^{\circ} 23^{\prime} \mathrm{O}\right)$. El clima de la región es templado (la temperatura media anual a $700 \mathrm{~m}$ s.n.m. es de $17.5^{\circ} \mathrm{C}$ ) y subhúmedo (precipitación media anual de $954 \mathrm{~mm}$, pero con variaciones interanuales considerables entre 611 y $1506 \mathrm{~mm}$, oscilación ocurrida durante el período 1960 - 2006, J. J. Filardo, comunicación personal). En el paisaje predominan rocas del basamento plutónico metamórfico, cubiertas en algunos sectores por remanentes de rocas sedimentarias cretácicas y terciarias (Cioccale, 1999). La ladera oriental está integrada por diferentes unidades geomorfológicas escalonadas, se distinguen dos elementos fundamentales: los relictos de una antigua superficie de erosión en su parte cumbral, y un escarpe erosionado que la limita. El paisaje de la superficie cumbral, ubicado entre 1125 y 1500 m s.n.m. con una altura promedio de 1200 $\mathrm{m}$, está dominado por un relieve suave ondulado con inclinación general hacia el este, formado por lomas y colinas con pendientes rectas y convexas y valles abiertos con fondos planos. El escarpe erosionado se desarrolla entre 750 y 1125 m s.n.m., y comprende las morfologías más abruptas del sistema, con pendientes muy fuertes que alcanzan valores entre 35 y $50 \%$ (Cioccale, 1999). La vegetación está compuesta por un mosaico de pastizales, arbustales, bosques y comunidades de afloramientos rocosos, sujetos a presiones de pastoreo variables e incendios recurrentes (Gavier $\&$ Bucher, 2004). A lo largo del gradiente altitudinal se puede observar un predominio de bosques y matorrales en la zona baja, seguido por pastizales y afloramientos rocosos en el sector superior. La zona de transición entre bosques y pastizales se encuentra entre los 900 y 1100 m s.n.m. y, en líneas generales coincide con la zona de transición entre el escarpe y la zona cumbral. Es importante destacar que en esta franja altitudinal se encuentra un mosaico de todas las diferentes fisonomías mencionadas.

\section{Selección de sitios}

A través de un trabajo en conjunto con Defensa Civil de la Localidad de Río Ceballos, se recopilaron los registros de incendios en el área de estudio desde el año 1991 hasta el año 2007. Con esta información, sumada a consultas a los vecinos de la zona se seleccionaron, en el invierno de 2007, 35 sitios, repartidos en lugares con cuatro edades diferentes post-fuego: (1) "Dos años": ubicados en un área donde el último incendio ocurrió en el invierno de 2005, dos años antes de las mediciones realizadas para el presente estudio; además esta área ya se había quemado en el año 1997; (2) "Cuatro años", en un área donde el último incendio ocurrió en el año 2003; (3) "10-17 años", donde el último incendio ocurrió entre el año 1990 y 1997. Si bien el área donde se encuentran estos últimos sitios muestra marcas de fuego, no se pudo saber con exactitud la fecha del último incendio; sin embargo, se estima que fue entre el año 1990 y 1997, por información suministrada por los pobladores; (4) "sin quemar", estos sitios carecen de marcas de fuego, y no hay registros de incendios por parte de los pobladores, por lo que se estima que no se queman hace por lo menos 60 años, o aun más. A diferencia de las otras tres edades, donde los sitios se encontraban asociados espacialmente, los sitios sin quemar se distribuyeron en toda el área de estudio. Todos los sitios están destinados a la producción ganadera desde el siglo XVII (Page, 2005).

Para cada una de las edades post-fuego, los sitios seleccionados estuvieron repartidos en todas las combinaciones posibles de exposición de ladera (norte, sur y este/oeste), y posición topográfica (ladera alta, ladera media y ladera baja). Como no se encontró un sitio sin quemar con exposición sur y en posición de ladera alta, el total de sitios seleccionados fue de 35 (4 edades x 3 exposiciones x 3 posiciones $-1=35$ ). Las laderas norte son las que reciben mayor insolación durante el año, mientras que las laderas sur son las que reciben menor radiación. Las laderas este y oeste se consideraron indistintamente, ya que la 


\section{A. Giorgis et al. - Fuego y topografía en zona de transición de bosques y pastizales}

insolación recibida a lo largo del año es similar, y numerosos antecedentes indican que la variación de la vegetación entre laderas norte y sur es mayor que las observadas entre laderas este y oeste, tanto en el área de estudio como en otros lugares a latitudes similares o mayores (Montaña \& Ezcurra, 1982; Bertrán de Solís, 1986; Anchorena \& Cingolani, 2002; Cingolani et al., 2008).

Entre enero y marzo de 2008 se registró la lista florística completa de plantas vasculares y se estimó visualmente el porcentaje de cobertura de cada especie (desde: 0,05 (menores a $1 \%$ ), 1, 2, 3, 5, 8, 10 , y desde aquí con rangos de incrementos cada 5 $\%$ hasta $100 \%$ ), en parcelas de $20 \times 20$ m ubicadas en cada uno de los 35 sitios. Además se estimó la cobertura de los estratos herbáceo, arbustivo y arbóreo. La diferenciación entre los estratos arbustivo y arbóreo se realizó en base a la altura de las especies leñosas (límite entre 2,5 y $3 \mathrm{~m}$ de altura independientemente de la identidad de las mismas). Para la nomenclatura botánica de la mayor parte de las especies registradas seguimos a Zuloaga et al., (2008), y su actualización "on line" (www.darwin. edu.ar). Para el género Gymnocalycium, se siguió a Demaio et al. (2011), y para Bidens y Gnaphalium a Ariza (2000 y 2008, respectivamente). Además se recolecto en cada sitio una muestra compuesta de suelo (resultante de 5 sub-muestras de suelo distribuidas al azar dentro del sitio) que fue sometida a los siguientes análisis: granulometría (Day 1986), pH (Thomas, 1996), materia orgánica (\%) mediante la técnica Walkley-Black (Nelson \& Sommers, 1996), nitrógeno total (\%) mediante la técnica Kjeldahl (Bremner, 1996), fósforo extractable (ppm) mediante la técnica Bray \& Kurtz $\mathrm{N}^{\circ} 1$ (Kuo, 1996), N-NO ${ }_{3}^{-}(\mathrm{ppm})$ y $\mathrm{N}_{-} \mathrm{NH}_{4}^{+}$(ppm) por potenciometría directa (Mulvaney, 1996), capacidad de intercambio catiónico (me/100g) y cationes $\left(\mathrm{Ca}^{++}, \mathrm{Mg}^{++}\right.$y K $\left.{ }^{+}\right)$(Sumner \& Miller, 1996). Finalmente, en cada sitio se midió la altitud (m s.n.m) y la pendiente (\%) ya que estos factores pueden condicionar la respuesta de la vegetación y el suelo (Swanson et al., 1988; Collins et al., 2007; Sherman et al., 2008; Coop et al., 2010). La altitud fue medida con un sistema de posicionamiento global (GPS) y la pendiente a través de dos formas: a nivel local con un clinómetro (RECTA SA, CH2501), y a nivel de paisaje utilizando un modelo de elevación digital, considerando un radio de $315 \mathrm{~m}$ alrededor del sitio (para ello se empleó un modelo de elevación digital obtenido de http://srtm.csi. cgiar.org/).

\section{Análisis de datos}

Además de la identificación taxonómica, las especies fueron clasificadas según su forma de vida siguiendo a Giorgis et al., (2011a), dentro de las siguientes categorías: árboles, arbustos, hierbas perennes caducifolias y siempre-verdes, hierbas anuales, cactáceas globulares, cactáceas columnares, cactáceas opunteoides, epífitas, helechos, enredaderas, gramíneas perennes en mata, graminoides (ciperáceas, juncáceas y gramíneas tanto perennes como anuales que no crecen formando grandes matas) y parásitas. Teniendo en cuenta que la invasión por especies exóticas es un factor modulador de las características de la vegetación en las Sierras de Córdoba (Giorgis et al., 2011a, b y c), especialmente en el sector donde está incluida el área de estudio (Hoyos et al., 2010; Gavier-Pizarro et al., 2012), todas las especies fueron también clasificadas según su origen en exóticas y nativas. Se consideraron exóticas a todas las especies que son citadas como adventicias, introducidas, naturalizadas, o exóticas propiamente dichas en el catálogo de las Plantas Vasculares del Cono Sur (Zuloaga et al., 2008).

En el diseño del estudio no se controló específicamente por la altitud y la pendiente, pero son dos variables que pueden influir sobre las respuestas de la vegetación y el suelo. Por ello, para facilitar la interpretación de los resultados se analizó si estos factores se asociaban a la edad post-fuego, la exposición de la ladera y la posición topográfica (que son los factores que sí se controlaron). Se utilizaron tres ANOVAs a tres vías con la altitud y las dos pendientes (a nivel local y de paisaje) como variables dependientes y los tres factores mencionados como variables independientes. Para cada una de las variables dependientes se seleccionaron los factores que fueron significativos, y en los casos en los que hubo dos factores con efectos significativos se puso a prueba la interacción entre ellos. Para comparar la riqueza de especies en relación a los distintos factores (edad post-fuego, exposiciones de ladera y posición topográfica), se realizó un análisis de varianza a tres vías, incluyendo además la altitud y las pendientes (a nivel local y de paisaje) como co-variables. Se seleccionó el mejor conjunto 
de variables eliminando aquellas que no fueron significativas. Se evaluó la interacción entre las variables seleccionadas. Finalmente, para todos los modelos se comprobó la normalidad de los residuos a través de la prueba de normalidad (pruebas de Kolmogorov-Smirnov, Shapiro-Wilk) y homogeneidad de varianza (prueba de Levene). Para identificar las diferencias entre medias realizamos una Prueba de Tukey.

Para conocer la relación entre los 35 sitios según su fisonomía, composición florística y las propiedades de los suelos, se realizaron tres análisis de ordenación, utilizando el método de coordinadas principales (PCoA, Podani, 1994), considerando la distancia euclidiana entre las unidades de muestreo. Para el análisis de la fisonomía se utilizó una matriz de 35 sitios por 16 variables. Las variables fueron las coberturas de los tres estratos y de cada una de las formas de vida mencionadas arriba (obtenida sumando las coberturas de las especies componentes). Para la composición florística se uso una matriz de 35 sitios por 223 variables. Las variables fueron las coberturas de cada una de las especies transformadas a la escala propuesta por Braun-Blanquet y modificada por van der Maarel, (1979). Para este análisis se eliminaron las especies que aparecieron solamente en uno o dos sitios. Para el análisis de las propiedades de los suelos se utilizó una matriz de 35 sitios por 17 variables. En este análisis las variables fueron las distintas características edáficas analizadas: materia orgánica; carbono orgánico; nitrógeno total; relación $\mathrm{C}: \mathrm{N} ; \mathrm{N}_{-} \mathrm{NO}_{3}^{-} ; \mathrm{N}_{-} \mathrm{NH}_{4}^{+} ; \mathrm{P} ; \mathrm{pH}$; conductividad eléctrica; $\mathrm{Ca}^{2+} ; \mathrm{Mg}^{2+} ; \mathrm{Na}^{+} ; \mathrm{K}^{+}$; capacidad de intercambio catiónico; arena (\%); arcilla (\%) y limo (\%), que se estandarizaron a través del Gower index (Podani, 1999).

Para analizar el efecto de las cuatro situaciones con distinta edad post-fuego, exposición de ladera, posición topográfica y sus combinaciones, sobre la fisonomía, la composición florística y las propiedades del suelo, se realizaron respectivos análisis de permutaciones al azar (Pillar \& Orlóci, 1996; Pillar, 2004). Para estos análisis se emplearon las mismas matrices que se utilizaron para cada uno de los tres análisis de ordenamiento: el fisonómico ("sitios x estratos y formas de vida"), el florístico ("sitios x especies"), y el edáfico ("sitios x características edáficas"). El procedimiento se llevó a cabo mediante el programa Multiv 2.63b (Pillar,
2004). Además, se hicieron análisis de correlación de Pearson entre la altitud y la pendiente a nivel local y de paisaje, con los ejes 1 y 2 de los PCoA fisonómico, florístico y edáfico.

\section{Resultados}

En los 35 censos se registró un total de 322 especies de plantas vasculares, con un $10,6 \%$ de especies exóticas (34). De un total de 71 familias, las más ricas fueron las Asteraceae (60) y Poaceae (56). Las formas de vida mejor representadas fueron las hierbas perennes siempre-verdes (89), graminoides (46) y arbustos (42).

\section{Análisis de la altitud y la pendiente}

Aunque el rango altitudinal de variación entre los distintos sitios es relativamente pequeño (908 a $1128 \mathrm{~m}$ ), los sitios quemados hace 10-17 años se encontraron a una altitud significativamente mayor que los sitios de otras edades post-fuego, entre los cuales no hubo diferencias significativas (Fig. 1a). La altitud, en cambio, no varió significativamente entre las distintas exposiciones de ladera o posiciones topográficas (resultados no mostrados).

La pendiente a escala local varió entre 4 y 84 $\%$, encontrándose diferencias significativas entre los sitios con diferentes posiciones topográficas $(\mathrm{F}=3,77$ y $p=0,03)$, siendo mayor en la ladera baja, intermedia en ladera media y menor en ladera alta (Fig. 1b). No se encontraron diferencias significativas en la pendiente a escala local de los sitios de las distintas edades post-fuego y exposición de ladera (datos no mostrados). La pendiente a escala de paisaje varió entre 5 y 30 $\%$, y no se encontraron diferencias significativas entre las distintas edades post-fuego, exposición de ladera y posición topográfica.

\section{Análisis de la riqueza de especies}

La riqueza de especies fue muy variable, encontrándose un mínimo de 22 y un máximo de 106 especies por sitio. Sólo la edad postfuego y la posición topográfica tuvieron efectos significativos sobre la riqueza de especies $(\mathrm{F}=$ $9,71, p=<0,0001)$, sin encontrarse una interacción significativa entre ambos factores. Además, ninguna de las co-variables tuvo efectos significativos. La edad post-fuego determinó las diferencias más 

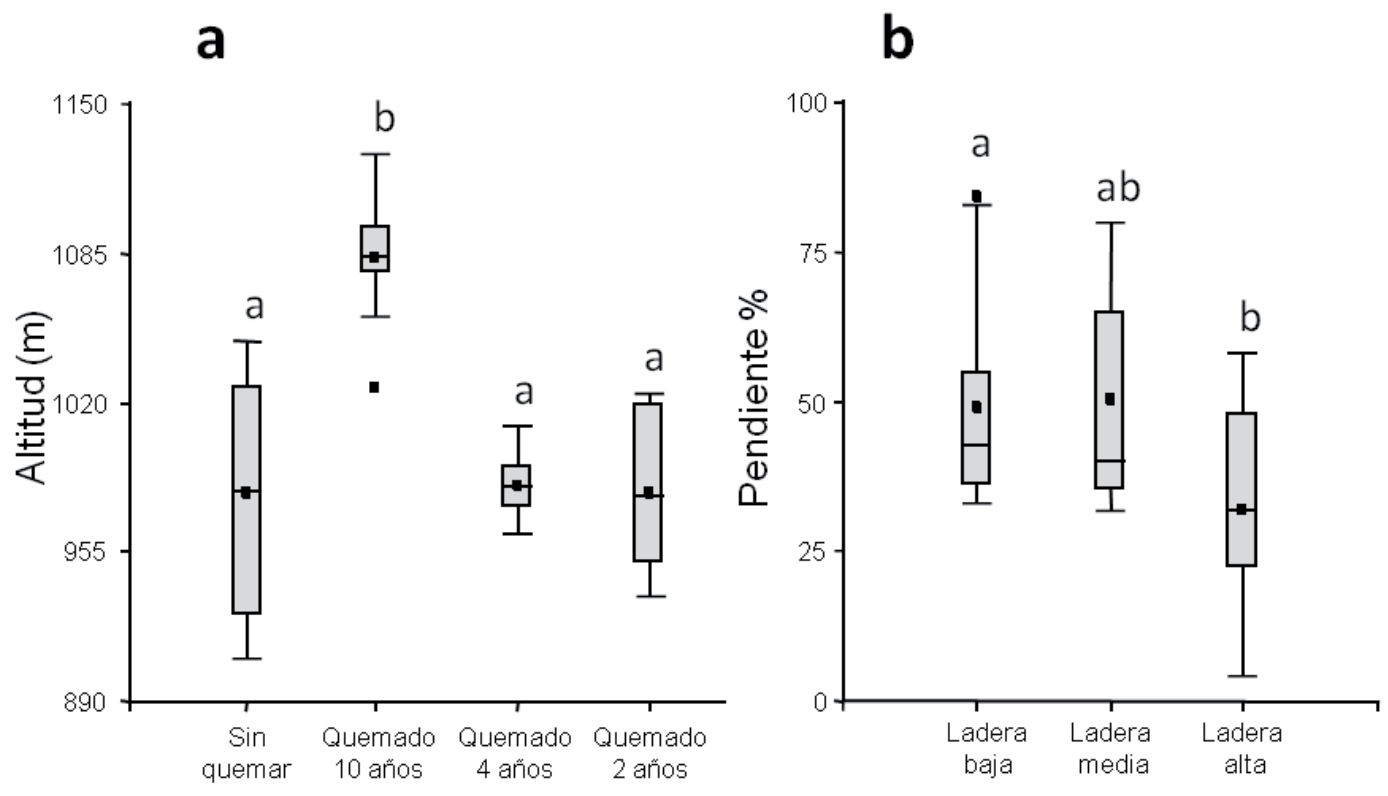

Fig. 1. Altitud de los sitios incluidos en cada una de las edades post-fuego (a), y pendiente local de los sitios incluidos en cada una de las distintas posiciones topográficas (b) en la zona de transición entre bosques y pastizales en las Sierras de Córdoba. Letras sobre las cajas indican diferencias significativas (ANOVA y test de Tukey, $p<0.05$ ).

importantes en la riqueza de especies $(\mathrm{F}=13,17$, $p=<0,0001)$. Los sitios sin quemar tuvieron un menor número de especies en relación a los sitios en las restantes edades post-fuego, entre los cuales no hubo diferencias significativas (Fig. 2a). La relación con la posición topográfica fue más débil $(\mathrm{F}=3,45, p=0,04)$. Los sitios en ladera media tuvieron el menor número de especies, mientras que los sitios en ladera alta registraron la mayor riqueza (Fig. 2b).

\section{Análisis de la fisonomía}

Los ejes 1 y 2 del ordenamiento explicaron respectivamente el 67,7 y el 13,8 \% de la variación en la fisonomía. El eje 1 mostró una variación desde bosques cerrados en el extremo negativo hacia pastizales o herbazales en el extremo positivo (Fig. 3a). Las variables que más contribuyeron de forma negativa con el eje 1 fueron la cobertura del estrato arbóreo, árboles y epífitas, y de forma positiva, la cobertura del estrato herbáceo y de las gramíneas perennes en mata. Las variables que más contribuyeron positivamente con el eje 2 fueron la cobertura de arbustos y la cobertura del estrato arbustivo; mientras que la variable que más contribuyó negativamente con ese eje fue la cobertura de gramíneas perennes en mata (Fig. 3a).

Los sitios sin quemar se separaron de los sitios de las restantes edades post-fuego en el eje 1 (Fig. $3 b)$, y se ubicaron en el extremo negativo del eje 1, indicando que en ellos predominan los árboles. Los restantes sitios se ubicaron en el extremo opuesto del mismo, mostrando una predominancia de arbustos y herbáceas (Fig. 3b). Si bien con respecto al eje 2 el patrón es menos evidente, se observó que los sitios quemados hace 10-17 años tienden a ubicarse en el extremo negativo del mismo, caracterizado por la dominancia de gramíneas perennes en mata. Los sitios quemados hace 4 años tendieron a ubicarse en el extremo positivo, caracterizado por un estrato arbustivo dominante y la cobertura de arbustos (Fig. 3b). Confirmando la observación visual en el plano del ordenamiento, el análisis de permutaciones al azar indicó que existen diferencias significativas entre los sitios sin quemar y cada una de las restantes edades post-fuego (Tabla 1). La mayor diferencia se encontró entre los sitios sin quemar y los quemados hace 10-17 años. También se encontraron diferencias 

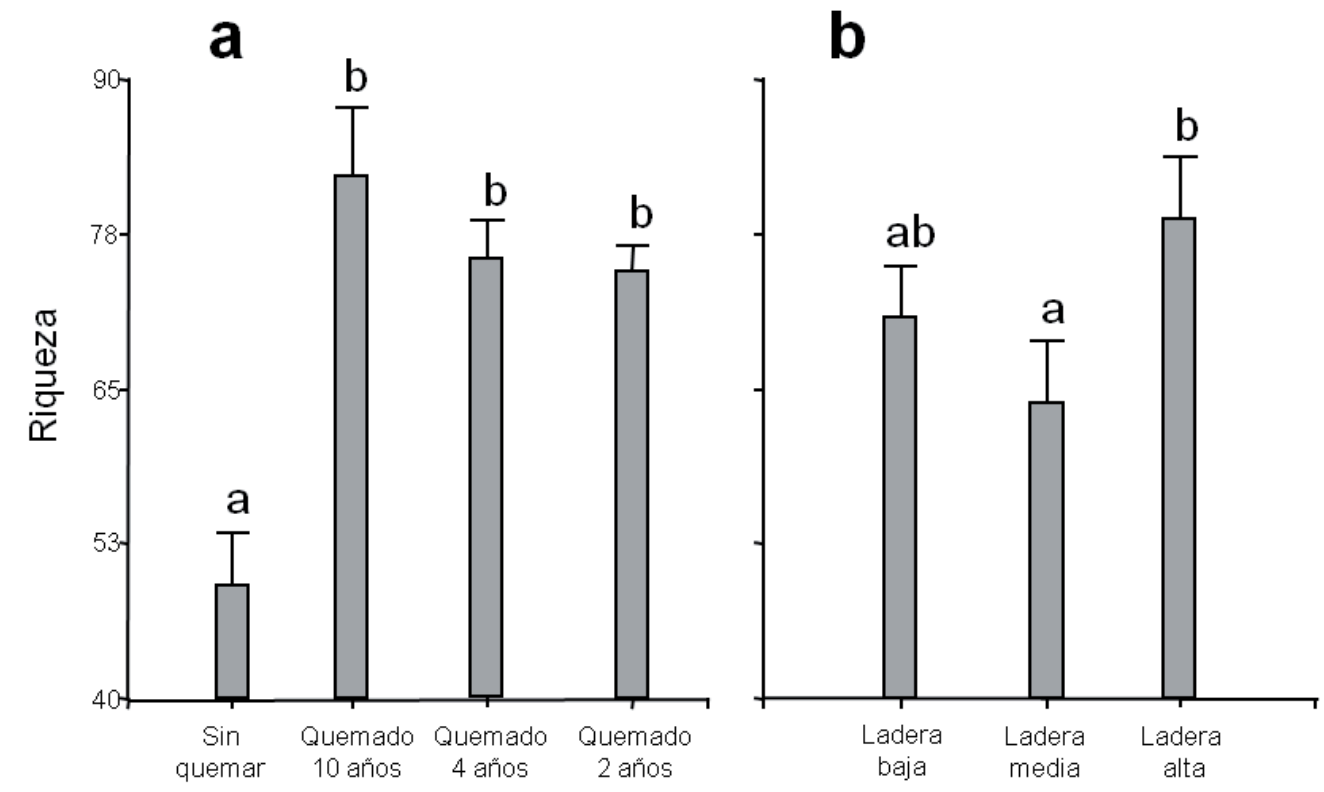

Fig. 2. Riqueza de especies para cada una de las edades post-fuego (a) y posición topográfica (b), en la zona de transición entre bosques y pastizales en las Sierras de Córdoba. Letras sobre las cajas indican diferencias significativas (ANOVA y test de Tukey, $p<0.05$ ).

significativas entre sitios quemados hace 10-17 años vs quemados hace 4 años (Tabla 1 ).

Con respecto a la exposición de la ladera sólo se observó una tendencia clara en el eje 2 del PCoA (Fig. 3c), donde los sitios con orientación sur tienden a ubicarse en el extremo negativo, definido por una alta cobertura de gramíneas perennes en mata. El análisis de permutaciones al azar confirmó este patrón, al indicar que las laderas con orientación sur difieren significativamente de las laderas con orientación norte y este/oeste (Tabla 1). En relación a la posición topográfica, se observó que cuatros sitios en ladera media y alta tienden a ubicarse en el sector negativo del eje 1 y positivo del eje 2 , con mayores coberturas de árboles y/o arbustos. El resto de los sitios se encontraron en el extremo positivo del eje $1 \mathrm{y}$ a lo largo de todo el eje 2, encontrándose que los sitios en ladera baja y media tienden a ubicarse en el extremo negativo de este eje, con mayor cobertura de gramíneas en mata (Fig. 3d). El análisis de permutaciones al azar sólo reflejó diferencias significativas entre sitios en ladera media vs ladera alta (Tabla 1). Además se encontraron interacciones significativas entre la edad post-fuego y la exposición de ladera y entre la edad post-fuego y la posición topográfica (Tabla 1), resultado que se observa al comparar la fig. $3 b$ vs $3 c$ y $3 b$ vs $3 d$, respectivamente.

Finalmente sólo el eje 1 del PCoA se correlacionó negativa y significativamente con la pendiente del paisaje ( $\mathrm{r}=-0,33, p=0,05)$, encontrándose que en general los sitios ubicados en el extremo negativo del eje 1, con altas coberturas del estrato arbóreo, tienen pendientes altas.

\section{Análisis de la composición florística}

Los ejes 1 y 2 del ordenamiento explicaron respectivamente el 27,7 y el $11,9 \%$ de la variación en composición florística. Las especies que más contribuyeron de forma negativa con el eje 1 fueron Mandevilla pentlandiana (A. DC.) Woodson, Eupatorium viscidum Hook. \& Arn., Setaria oblongata (Griseb.) Parodi y Celtis ehrenbergiana (Klotzsch) Liebm. Las especies que más contribuyeron de forma positiva fueron Festuca hieronymi Hack, Krapovickasia flavescens (Cav.) Fryxell y Piptochaetium montevidense (Spreng.) Parodi (Fig. 4a). Con el eje 2 contribuyeron de forma negativa Jarava pseudoichu (Caro) F. Rojas, Acacia caven (Molina) Molina, Acalypha communis 


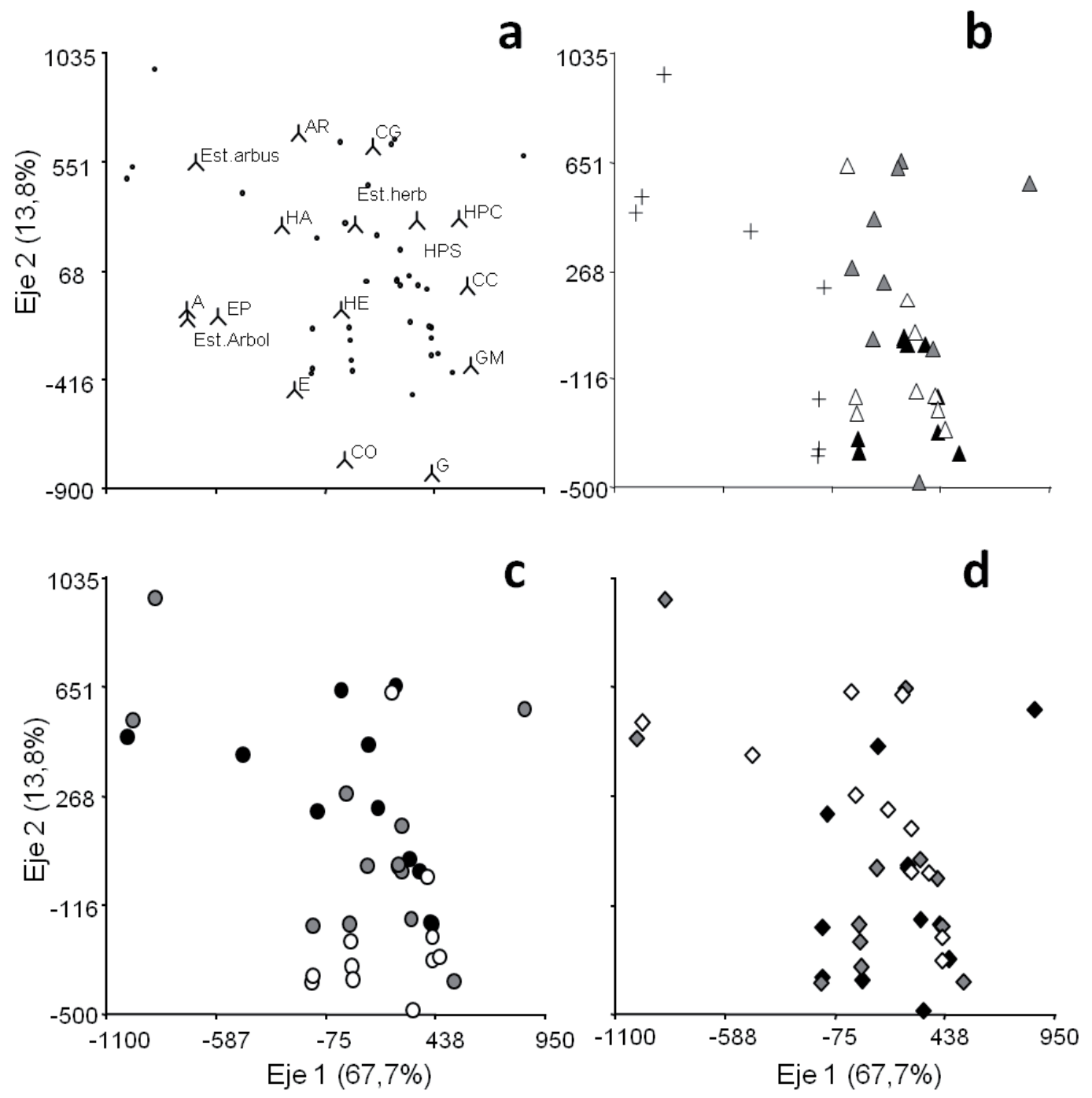

Fig. 3. Ordenación en el plano definido por los ejes 1 y 2 de PCoA de los 35 censos realizados en la zona de transición entre bosques y pastizales, en base a la fisonomía de la vegetación. (a) Los distintos censos (puntos) según su fisonomía, se grafican las variables (入); árboles (A), arbustos (AR), hierbas perennes caducifolias (HPC) y siempre-verdes (HPS), hierbas anuales (HA), cactáceas globulares (CG), cactáceas columnares (CC), cactáceas opunteoides (CO), epifitas (EP), gramíneas perennes en mata $(\mathrm{GM})$, graminoides $(\mathrm{G})$, enredaderas $(\mathrm{E})$, helechos $(\mathrm{HE})$, estrato arbóreo (Est.Arbol), estrato arbustivo (Est. arbus), estrato herbáceo (Est.herb). (b) Censos según edades post-fuego: sin quemar $(\uparrow)$, quemado hace 10-17 años $(\boldsymbol{\Delta})$, quemado hace 4 años $(\Delta)$ y quemado hace 2 años $(\Delta)$. c) Censos según exposición: norte $(\boldsymbol{)})$, este/oeste $(\mathrm{O})$ y sur $(\mathrm{O})$. (d) Censos según posición topográfica: ladera baja $(\diamond)$, ladera media $(\diamond)$ y ladera alta $(\diamond)$.

Müll. Arg. y Gaya parviflora (Phil.) Krapov. En cambio, contribuyeron de forma positiva: Woodsia montevidensis (Spreng.) Hieron. y Anemia australis (Mickel) M. Kessler \& A.R. Sm. (Fig. 4a).
En el extremo negativo del eje 1 se ubicaron los sitios sin quemar mientras que en el extremo positivo se agruparon los sitios quemados hace 10-17 años (Fig. 4b). Los sitios de las otras dos 
Tabla 1. Efectos de las edades post-fuego,

la exposición, la posición topográfica y sus combinaciones sobre la fisonomía de la vegetación en la zona de transición entre bosques y pastizales en las Sierras de Córdoba. Las relaciones se expresan a través del Test de Permutaciones al azar.

\begin{tabular}{|c|c|c|}
\hline Efectos y contrastes & $\begin{array}{l}\text { Suma de } \\
\text { cuadrados }\end{array}$ & $p$ \\
\hline Factor edad post-fuego & 125000 & 0,0002 \\
\hline $\begin{array}{l}\text { Sitios no quemados vs } \\
\text { quemados hace } 10-17\end{array}$ & 89834 & 0,003 \\
\hline $\begin{array}{l}\text { Sitios no quemados vs } \\
\text { quemados hace } 4\end{array}$ & 68961 & 0,006 \\
\hline $\begin{array}{l}\text { Sitios no quemados vs } \\
\text { quemados hace } 2\end{array}$ & 83069 & 0,004 \\
\hline Quemado hace $10-17$ vs hace 4 & 8112 & 0,006 \\
\hline Quemado hace $10-17$ vs hace 2 & 1984,6 & 0,308 \\
\hline Quemado hace 4 vs hace 2 & 4533,8 & 0,055 \\
\hline Exposición de ladera & 12290 & 0,0002 \\
\hline Norte vs Este/oeste & 1893,4 & 0,183 \\
\hline Norte vs Sur & 10693 & 0,0006 \\
\hline Este/oeste vs Sur & 6032,3 & 0,004 \\
\hline Posición Topográfica & 6865,5 & 0,076 \\
\hline Ladera baja vs Ladera media & 1248,7 & 0,545 \\
\hline Ladera baja vs Ladera alta & 4418,3 & 0,125 \\
\hline Ladera media vs ladera alta & 4726,1 & 0,02 \\
\hline $\begin{array}{l}\text { Edad post-fuego } \\
\text { vs exposición }\end{array}$ & 13560 & 0,01 \\
\hline $\begin{array}{l}\text { Edad post-fuego vs } \\
\text { posición topográfica }\end{array}$ & 15689 & 0,008 \\
\hline $\begin{array}{l}\text { Exposición vs posición } \\
\text { topográfica }\end{array}$ & 5054,5 & 0,9948 \\
\hline $\begin{array}{l}\text { Edad post-fuego vs } \\
\text { Expo. vs Pos. Top. }\end{array}$ & 9540,8 & 0,9248 \\
\hline
\end{tabular}

* En negrita se destacan los valores significativos.

edades post-fuego se distribuyeron tanto en el centro como en el extremo positivo del eje 1. El eje 2 agrupó los sitios sin quemar y los quemados hace 10-17 años en el extremo positivo, mientras que los restantes sitios se hallaron distribuidos a lo largo de todo el eje (Fig. 4b). El análisis de permutaciones reflejó que las diferencias más importantes en composición florística se encontraron entre sitios sin quemar y sitios quemados hace 10-17 años (Tabla 2). Los sitios sin quemar también difirieron significativamente de las restantes edades postfuego. Además fueron significativas las diferencias florísticas entre los sitios quemados hace 10-17 años vs quemados hace 4 y 2 años, y entre sitios quemados hace 4 y 2 años, siendo más importantes las diferencias entre los sitios quemados hace 10-17 años vs quemados hace 4 y 2 años (Tabla 2).

Con respecto a la exposición de ladera, observamos un patrón asociado al eje 2 (Fig. 4c), encontrándose los sitios con exposición sur ubicados en el extremo positivo del eje, mientras que las otras exposiciones se disponen hacia el extremo negativo del mismo. En el análisis de permutaciones se encontraron diferencias significativas entre las tres exposiciones, siendo más marcada entre sitios con exposición norte vs sur (Tabla 2). Entre las distintas posiciones topográficas se observó una leve diferencia a lo largo del eje 2, encontrándose los sitios en ladera alta generalmente agrupados en el sector negativo, mientras que los sitios en ladera media y baja muestran una tendencia opuesta (Fig. 4d). Estas tendencias fueron confirmadas estadísticamente por el análisis de permutaciones al azar (Tabla 2). Sólo la interacción entre la edad post-fuego y la exposición mostró un efecto significativo (Tabla 2): en general, los sitios recientemente quemados, que se encuentran ubicados en el extremo positivo del eje 1 y 2 , tienen exposición sur (Fig. 4 b y c).

Al correlacionar la altitud y las dos pendientes con el eje 1 y 2 del PCoA con datos florísticos, se encontró que la altitud se correlacionó positiva y significativamente con el eje $1(\mathrm{r}=0,42$ y $p=0,01)$, reflejando que los sitios quemados hace 10-17 años, que tienden a ubicarse en el extremo positivo del eje 1, están a mayor altitud. Finalmente, la pendiente a escala del paisaje se encontró positiva y significativamente correlacionada con el eje 2 del ordenamiento florístico $(\mathrm{r}=0,34$ y $p=0,04)$.

\section{Análisis de las propiedades del suelo}

Los ejes 1 y 2 del ordenamiento explicaron respectivamente el 24,37 y el $12,2 \%$ de la variación en las propiedades de los suelos (Fig. 5a). Las variables que más contribuyeron de forma negativa con el eje 1 fueron la capacidad de intercambio catiónico, el contenido de calcio y el porcentaje de materia orgánica y carbono; el contenido de arena contribuyó positivamente a la variación en ese eje (Fig. 5a). A lo largo del eje 2 contribuyeron negativamente el contenido de limo y magnesio 


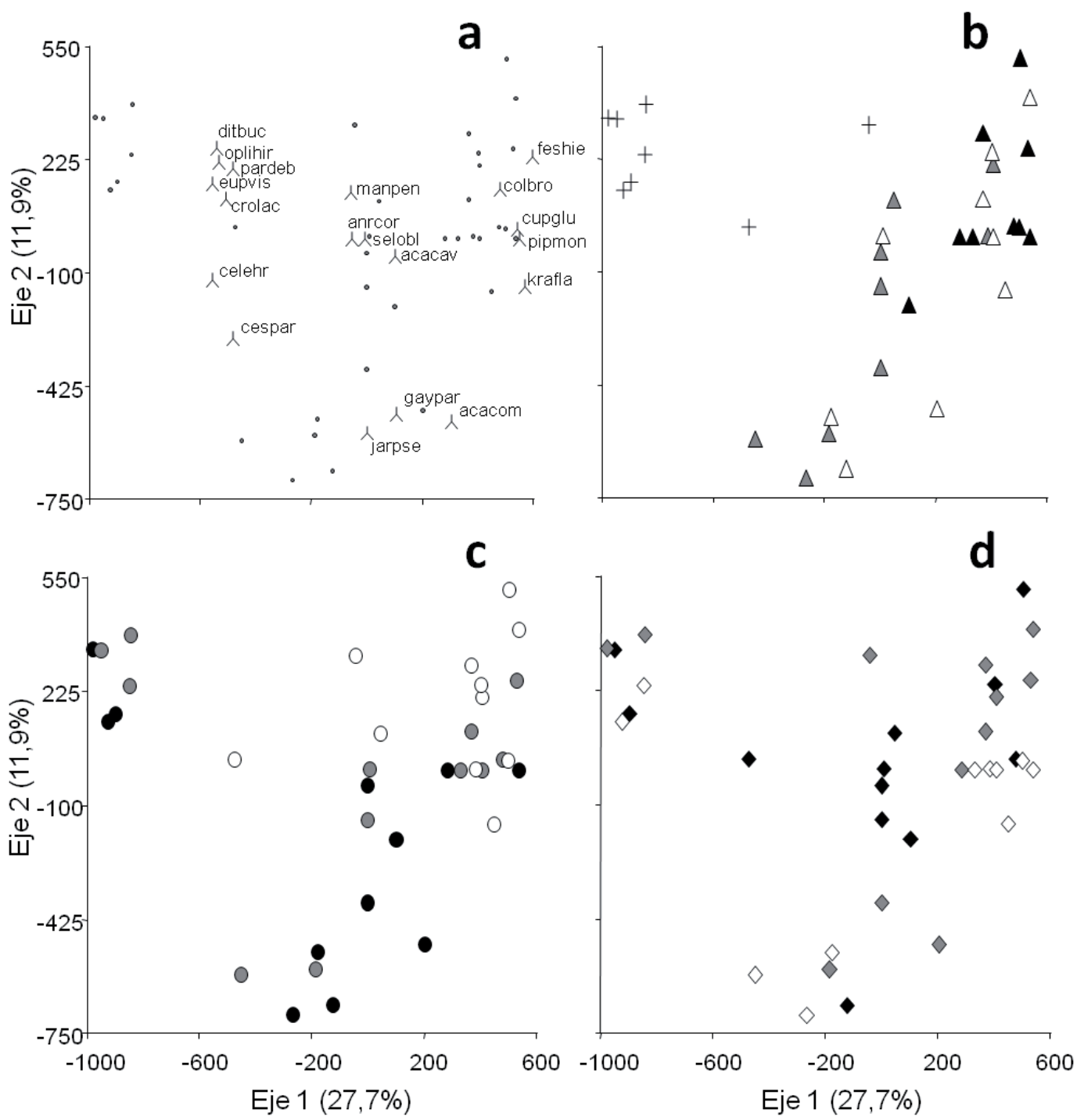

Fig. 4. Ordenación de los 35 censos realizados en la zona de transición entre bosques y pastizales en las Sierras de Córdoba; se grafican el eje 1 y el eje 2 generados a partir de PCoA realizado en base a la composición florística. (a): ubicación de los distintos sitios (puntos) según su comunidad florística; se representan las variables ( $($ ) con una correlación mayor a 0,7 con los ejes 1 o 2; Eupatorium viscidum (eupvis), Celtis ehrenbergiana (celehr), Mandevilla pentlandiana (manpen), Setaria oblongata (setobl), Festuca hieronymi (feshie), Krapovickasia flavescens (krafla), Jarava pseudoichu (jarpse), Acacia caven (acacav), Acalypha communis (acacom), Gaya parviflora (gaypar), Anredera cordifolia (anrcor), Ditassa burchellii (ditbur), Oplismenus hirtelus (oplhir), Croton lachnostachyus (crolac), Cestrum parqui (cespar), Parietaria debilis (pardeb), Cologamia broussonetii (colbro), Cuphea glutinosa (cupglu), Piptochaetium montevidensis (pipmon). (b) Censos según edades post-fuego: sin quemar $(\rightarrow)$, quemado hace 10-17 años $(\boldsymbol{\Delta})$, quemado hace 4 años $(\Delta)$ y quemado hace 2 años $(\Delta)$. (c) Censos según exposición: norte $(\mathbf{O})$, este/oeste $(0)$ y sur $(\mathrm{O})$. (d) Censos según posición topográfica: ladera baja $(\diamond)$, ladera media $(\diamond)$ y ladera alta $(\diamond)$. 
Tabla 2. Efectos de las edades post-fuego,

la exposición, la posición topográfica y sus

combinaciones, sobre la composición florística en

la zona de transición entre bosques y pastizales

en las Sierras de Córdoba. Las relaciones se

expresan a través del Test de Permutaciones al

\section{azar.}

\begin{tabular}{|c|c|c|}
\hline Efectos y contrastes & $\begin{array}{l}\text { Suma de } \\
\text { cuadrados }\end{array}$ & $p$ \\
\hline Factor edad post-fuego & 1078,4 & 0,0002 \\
\hline $\begin{array}{l}\text { Sitios no quemados vs } \\
\text { quemados hace } 10-17\end{array}$ & 650,39 & 0,004 \\
\hline $\begin{array}{l}\text { Sitios no quemados vs } \\
\text { quemados hace } 4\end{array}$ & 475,92 & 0,005 \\
\hline $\begin{array}{l}\text { Sitios no quemados vs } \\
\text { quemados hace } 2\end{array}$ & 553,5 & 0,005 \\
\hline Quemado hace $10-17$ vs hace 4 & 228 & 0,004 \\
\hline Quemado hace $10-17$ vs hace 2 & 154,22 & 0,004 \\
\hline Quemado hace 4 vs hace 2 & 128,11 & 0,022 \\
\hline Exposición de ladera & 406,63 & 0,0002 \\
\hline Norte vs Este/oeste & 110,96 & 0,009 \\
\hline Norte vs Sur & 320,6 & 0,001 \\
\hline Este/oeste vs Sur & 182,21 & 0,001 \\
\hline Posición Topográfica & 170,45 & 0,011 \\
\hline Ladera baja vs Ladera media & 66,46 & 0,28 \\
\hline Ladera baja vs Ladera alta & 101,64 & 0,0266 \\
\hline Ladera media vs ladera alta & 88,39 & 0,0072 \\
\hline Edad post-fuego vs exposición & 489,59 & 0,0002 \\
\hline $\begin{array}{l}\text { Edad post-fuego vs } \\
\text { posición topográfica }\end{array}$ & 441,44 & 0,1118 \\
\hline $\begin{array}{l}\text { Exposición vs posición } \\
\text { topográfica }\end{array}$ & 242,08 & 0,9728 \\
\hline $\begin{array}{l}\text { Edad post-fuego vs } \\
\text { Expo. vs Pos. Top. }\end{array}$ & 642,37 & 0,9808 \\
\hline
\end{tabular}

* En negrita se destacan los valores significativos.

y, positivamente, el contenido de arena y la conductividad eléctrica (Fig. 5a).

Se observó una clara separación entre los sitios sin quemar agrupados en el extremo negativo del eje $1 \mathrm{y}$ los sitios recientemente quemados ubicados en el extremo opuesto del eje 1 y negativo del eje 2 (Fig. $5 b)$. Esto indica que los sitios sin quemar tuvieron suelos con bajo contenido de arena, con mayor contenido de materia orgánica y de calcio y mayor capacidad de intercambio catiónico que los sitios de las restantes edades post-fuego. A través del análisis de permutaciones se confirmó que existen diferencias significativas entre sitios sin quemar y cada una de las restantes edades post-fuego analizadas (Tabla 4). Es importante destacar que las diferencias más grandes
Tabla 3. Efectos de la edad post-fuego, la exposición, la posición topográfica y sus combinaciones sobre las propiedades del suelo en la zona de transición entre bosques y pastizales en las Sierras de Córdoba. Las relaciones se expresan a través del Test de Permutaciones al azar.

\begin{tabular}{|c|c|c|}
\hline Efectos y contrastes & $\begin{array}{l}\text { Suma de } \\
\text { cuadrados }\end{array}$ & $p$ \\
\hline Factor edad post-fuego & 19,247 & 0,0002 \\
\hline $\begin{array}{l}\text { Sitios no quemados vs } \\
\text { quemados hace } 10-17\end{array}$ & 10,771 & 0,005 \\
\hline $\begin{array}{l}\text { Sitios no quemados vs } \\
\text { quemados hace } 4\end{array}$ & 0,875 & 0,008 \\
\hline $\begin{array}{l}\text { Sitios no quemados vs } \\
\text { quemados hace } 2\end{array}$ & 0,925 & 0,008 \\
\hline Quemado hace $10-17$ vs hace 4 & 0,397 & 0,009 \\
\hline Quemado hace $10-17$ vs hace 2 & 0,356 & 0,006 \\
\hline Quemado hace 4 vs hace 2 & 0,272 & 0,182 \\
\hline Exposición de ladera & 0,631 & 0,005 \\
\hline Norte vs Este/oeste & 0,331 & 0,025 \\
\hline Norte vs Sur & 0,402 & 0,013 \\
\hline Este/oeste vs Sur & 0,211 & 0,2 \\
\hline Posición Topográfica & 0,428 & 0,467 \\
\hline Ladera baja vs Ladera media & 0,219 & 0,385 \\
\hline Ladera baja vs Ladera alta & 0,169 & 0,752 \\
\hline Ladera media vs ladera alta & 0,253 & 0,248 \\
\hline Edad post-fuego vs exposición & 10,685 & 0,536 \\
\hline $\begin{array}{l}\text { Edad post-fuego vs } \\
\text { posición topográfica }\end{array}$ & 17,894 & 0,001 \\
\hline $\begin{array}{l}\text { Exposición vs posición } \\
\text { topográfica }\end{array}$ & 0,682 & 0,948 \\
\hline Fuego vs exp. vs pos. top. & 20,709 & 0,932 \\
\hline
\end{tabular}

* En negrita se destacan los valores significativos.

se encontraron entre los sitios sin quemar y los sitios quemados hace 10-17 años. Además, se encontraron diferencias significativas entre los sitios quemados hace 10-17 años y los sitios quemados hace 4 y 2 años, quienes no se diferenciaron significativamente entre sí (Tabla 4).

Con respecto a la exposición de la ladera, no se aprecia una clara tendencia en la Fig. 5c, aunque el análisis de permutaciones al azar detectó diferencias significativas entre laderas con exposición norte vs este/oeste y norte vs sur (Tabla 4). En cuanto a la posición topográfica tampoco se observa una clara separación en el análisis de Coordenadas Principales (Fig. 5d). En coincidencia, tampoco se encontraron efectos significativos de la posición topográfica sobre las propiedades del suelo (Tabla 4). Sólo el 

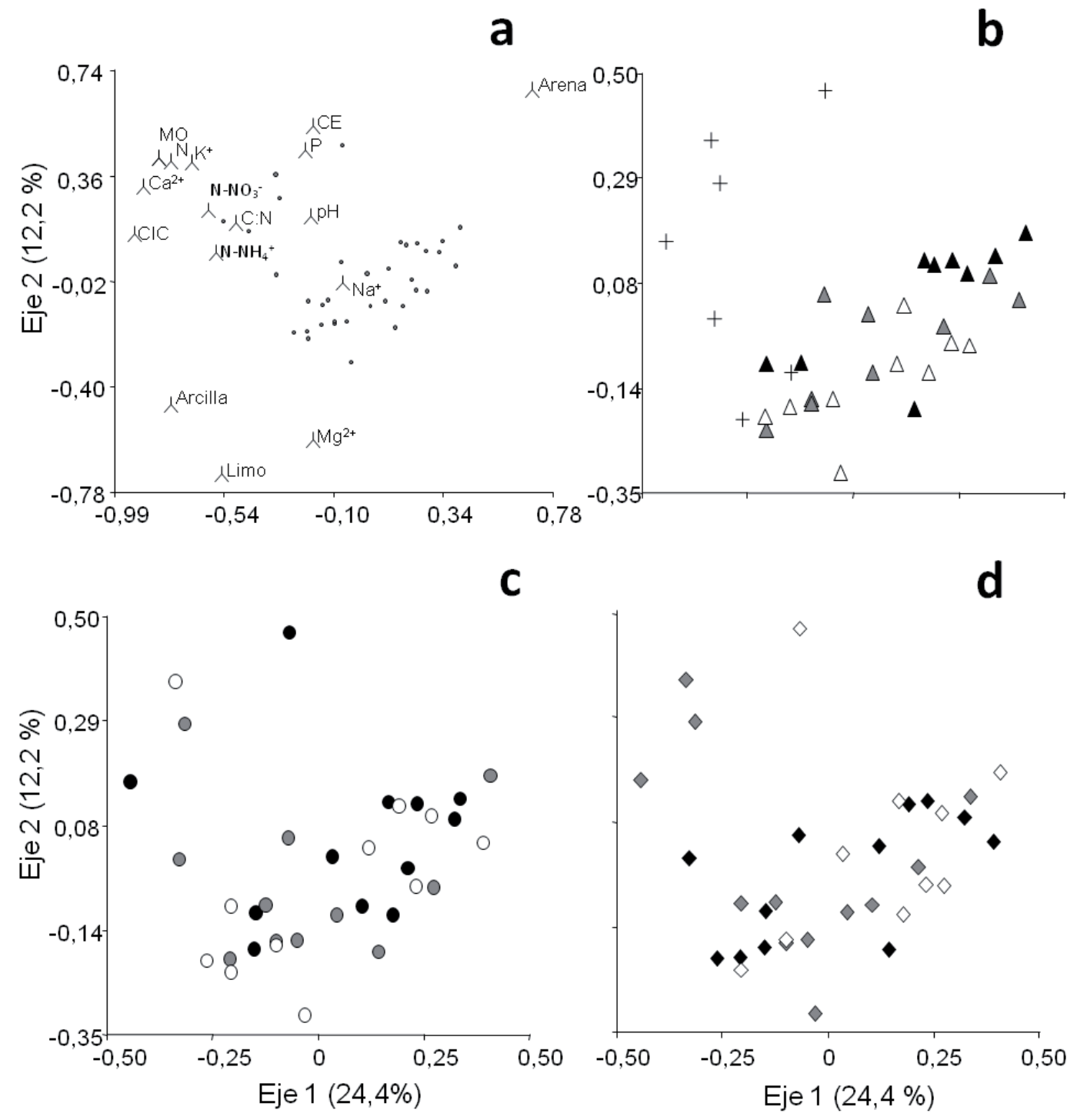

Fig. 5. Ordenación en el plano definido por los ejes 1 y 2 de PCoA de los 35 censos realizados en la zona de transición entre bosques y pastizales de las Sierras de Córdoba, en base a las propiedades del suelo. (a) Censos (puntos) dispuestos según las variables (入) edáficas: Materia orgánica (MO), Nitrógeno Total $(\mathrm{N})$, Relación C:N (C:N), Nitratos $\left(\mathrm{N}_{-} \mathrm{NO}_{3}{ }^{-}\right)$, nitritos $\left(\mathrm{N}-\mathrm{NH}_{4}^{+}\right)$, Carbono $(\mathrm{C})$, Fósforo $(\mathrm{P})$, Conductividad Eléctrica $(\mathrm{CE})$, Calcio $\left(\mathrm{Ca}^{2+}\right)$, Magnesio $\left(\mathrm{Mg}^{2+}\right)$, Sodio $(\mathrm{Na}+)$, Potasio $\left(\mathrm{K}^{+}\right)$, y capacidad de intercambio iónico $(\mathrm{CIC})$, Arena, Limo, Arcilla. (b) Censos según edades post-fuego: sin quemar ( $t)$, quemado hace 10-17 años $(\boldsymbol{\Lambda})$, quemado hace 4 años $(\triangle)$ y quemado hace 2 años $(\Delta)$. (c) Censos según exposición: norte $(\boldsymbol{O})$, este/oeste $(\mathrm{O})$ y sur $(\mathrm{O})$. (d) Censos según posición topográfica: ladera baja $(\diamond)$, ladera media $(\diamond)$ y ladera alta $(\diamond)$.

efecto combinado de la edad post-fuego y la posición topográfica tuvieron un efecto significativo (Tabla 4), situación que se puede observar en las Fig. 5b y $5 \mathrm{~d}$, donde sólo se segregaron con valores positivos en el eje 2 cuatro sitios sin quemar, con posición de ladera alta y media; el resto de los sitios sin quemar se encuentra junto con el resto de los sitios quemados en el extremo negativo del eje 2 . 
La altitud se encontró positivay significativamente correlacionada con los ejes 1 y 2 del ordenamiento basado en las propiedades del suelo $(\mathrm{r}=0,42$ y $p=$ 0,01 y $\mathrm{r}=0,35$ y $p=0,04$; respectivamente). En el primer caso esto se debe a que los sitios quemados hace 10-17 años (que tienen los suelos más pobres), se encontraron en el extremo positivo del eje 1 y a mayor altitud que el resto de los sitios. En el caso del eje 2 , se debe a que cuatro de los sitios sin quemar son los únicos que tuvieron valores altos en el eje 2 . Por otro lado, la pendiente a nivel local se encontró significativa y negativamente correlacionada con el eje 1 del ordenamiento en base a las propiedades del suelo $(\mathrm{r}=-0,4$ y $p=0,02)$. Los sitios con menores pendientes tienden a ubicarse en el extremo positivo del eje 1 , indicando que son suelos pobres con alto contenido de arena. La pendiente del paisaje también se encontró negativa y significativamente correlacionada con el eje 1 del ordenamiento de las propiedades del suelo $(\mathrm{r}=-0,36$ y $p=0,03)$. Algunos sitios con pendiente alta a nivel de paisaje tienden a ubicarse en el extremo negativo del eje 1, repitiéndose el patrón anteriormente mencionado para la pendiente a nivel local.

Es importante destacar que el patrón principal de los análisis de ordenación fue consistente con los resultados más importantes de los análisis de permutaciones al azar, para las características fisonómicas y florísticas de la vegetación y las propiedades de los suelos. A lo largo del eje 1 de los tres ordenamientos, los sitios sin quemar se segregaron en el extremo negativo, indicando que estos sitios son similares entre sí en cuanto a las tres características analizadas, mientras que los sitios recientemente quemados se distribuyeron separadamente a lo largo del extremo positivo del eje 1, en los tres casos. En general, en el eje 1 los sitios quemados hace 10-17 años se disponen en el extremo opuesto a los sitios sin quemar. También es importante destacar que el eje 1 de la composición florística y de las propiedades de los suelos se correlacionaron positivamente con la altitud.

\section{Discusıón}

De acuerdo con nuestras predicciones, los sitios con diferentes edades post-fuego presentaron mayores diferencias en las características de la vegetación y del suelo que sitios con distinta topografía pero similar edad post-fuego. Esto sugiere que el fuego es el principal factor estructurador de la vegetación y los suelos en la zona de transición entre el bosque y el pastizal en las Sierras de Córdoba. Resultados similares se han reportado para otras montañas de Argentina (Grau, 2003) y del mundo (Capitanio \& Carcaillet, 2008; Sherman et al., 2008; Carcaillet et al., 2009; Coop et al., 2010). También de acuerdo a lo esperado, los sitios que no experimentaron fuegos durante al menos los últimos 60 años presentaron una mayor cobertura arbórea y menor cobertura herbácea. Esto es también coincidente con resultados obtenidos en otras zonas montañosas de transición entre bosques y pastizales del mundo (por ejemplo: Grau, 2003, 2005; Behling \& Pillar, 2007; Coop et al., 2010). Sin embargo, sorprende que los sitios quemados hace 10-17 años, con el mayor tiempo transcurrido desde el último fuego, fueron los que más difirieron con respecto a los sitios sin quemar, tanto en su fisonomía y composición florística, como en las propiedades de los suelos. Estos sitios presentaron las menores coberturas arbóreas, y estuvieron caracterizados por una fisonomía de pastizal dominada por la gramínea perenne en mata Festuca hieronymi. Además, tuvieron los suelos con menos nutrientes de todas las situaciones estudiadas. En contraste, los sitios quemados hace 4 y 2 años fueron más parecidos, en los aspectos mencionados, a los sitios sin quemar. Se caracterizaron por tener una fisonomía arbustiva, dominados por especies leñosas como Acacia caven y Colletia spinossisima J. F. Gmel., presentando además suelos más ricos en nutrientes que los sitios quemados hace 10-17 años. En parte estas diferencias podrían deberse a que los sitios quemados hace 10-17 años se encuentran a mayor altitud y asociados a menores pendientes que el resto de los sitios. Diversos autores han sugerido que la falta de árboles en las partes más altas de las Sierras de Córdoba, y específicamente en el área de estudio, estaría relacionada a cambios climáticos vinculados a la altitud, la pendiente y la exposición de las laderas (Montaña 1975; Luti et al., 1979; Montaña \& Ezcurra, 1982). Sin embargo, y dado que el sitio de estudio se encuentra muy por debajo del límite térmico encontrado a nivel global para el crecimiento de árboles en montañas (Körner \& Paulsen, 2004, cf. Marcora et al., 2008), es poco probable que la disminución de la temperatura con la altitud sea el factor determinante 


\section{A. Giorgis et al. - Fuego y topografía en zona de transición de bosques y pastizales}

del límite de vegetación leñosa en nuestro caso. Además, la diferencia altitudinal entre sitios sin árboles y con árboles es menor a 100 metros. En consecuencia, sugerimos que para nuestros sitios de estudio, las diferencias climáticas asociadas a cambios en altitud y pendiente serían demasiado pequeñas como para transformarse en el principal factor en condicionar el establecimiento de especies leñosas o determinar las características del suelo. Por otra parte, en distintos lugares de las sierras se han observado individuos establecidos de especies leñosas nativas del Bosque Serrano hasta los $1750 \mathrm{~m}$ s.n.m. (Giorgis et al., 2011a), y evidencias recientes sugieren que no hay limitaciones climáticas para el establecimiento y crecimiento de especies leñosas nativas a lo largo de todo el gradiente altitudinal de las Sierras de Córdoba (Pais Bosch et al., 2012; Marcora et al., 2013).

Estudios recientes en distintos lugares del mundo muestran que los cambios en la vegetación $\mathrm{y}$, particularmente, en la presencia de árboles asociados con la altitud o la pendiente, se encuentran condicionados por la interacción entre factores antrópicos y variables climáticas (Grau, 2003; Cairns \& Moen, 2004; Behling \& Pillar, 2007; Cierjacks et al., 2007; Coop et al., 2010). En este contexto, es posible suponer que la falta de especies leñosas en los sitios quemados hace 10-17 años en las Sierras de Córdoba, se deba más bien al efecto conjunto de factores relacionados con la historia de disturbios, interactuando con las características ambientales propias del lugar, que al efecto exclusivo de un único factor. Por un lado, la herbivoría ha sido reportada como un factor limitante del crecimiento y establecimiento de plántulas de leñosas en distintos lugares del mundo (Kramer et al., 2003; Pillar, 2003; Cairns \& Moen, 2004; Cierjacks et al., 2007; Speed et al., 2010) y también en los sectores de las Sierras de Córdoba (Teich et al., 2005; Giorgis et al., 2010; Torres, 2012). En este sentido, una vez que el fuego transforma la estructura arbórea del bosque en una comunidad abierta y más baja, las especies quedan expuestas a la acción directa de los herbívoros, quienes pueden retrasar o impedir la recuperación de las especies leñosas (Cairns \& Moen, 2004). Por otro lado, la historia previa de recurrencia de fuegos puede estar condicionando fuertemente la recuperación post-fuego, limitando la capacidad de rebrote y la presión de propágulos en cada uno de los sitios (Chazdon, 2003; Sibold et al., 2006; Kitzberger et al., 2007; Coop et al., 2010). La frecuencia de los incendios está relacionada con las características de la vegetación preexistente, de manera que materiales finos se queman más fácilmente que materiales más gruesos (Albanesi \& Anriquez, 2004). En el área de estudio las principales comunidades que se incendian y propagan los fuegos son los pastizales (Carignano \& Cioccale, 1992; Miglietta, 1994, Giorgis M. A., datos no publicados), fisonomía dominante en el sector superior de las sierras y característica de los sitios quemados hace 10-17 años. Además, las características geomorfológicas del paisaje pueden condicionar la frecuencia y propagación de los incendios (Gibson \& Hulbert, 1987; Swanson et al., 1988; Turner \& Romme, 1994; Kramer et al., 2001). En este sentido, la zona cumbral de las sierras cuyo paisaje se encuentra dominado por pastizales de $F$. hieronymi, se caracteriza por tener relieves planos ondulados con una pendiente baja (Cioccale, 1999), encontrándose más expuestos a los vientos, a los fuegos y a la acción ganadera, que la zona del escarpe. En nuestros resultados esta relación se observa en la correlación positiva de la altitud y el eje 1 del ordenamiento florístico separando, en el extremo positivo, los sitios quemados hace 10-17 años y en la correlación negativa del eje 1 del ordenamiento fisonómico con la pendiente del paisaje, separando en el extremo negativo sitios con altas coberturas del estrato arbóreo. Asimismo, en la asociación positiva del eje 2 del ordenamiento florístico con la pendiente del paisaje separando, en el extremo positivo, los sitios quemados hace 10-17 años de los sitios quemados hace 4 y 2 años. En resumen, la ganadería, la historia previa de uso y las características topográficas asociadas a cambios ambientales en conjunto, podrían estar explicando la alta frecuencia de incendios en la zona cumbral y manteniendo la predominancia de pastizales en este sector de las sierras. En este contexto, sería muy importante conocer en más detalle el efecto conjunto de estos factores, ya que la co-ocurrencia de los mismos podría estar limitando la regeneración de las comunidades boscosas en las Sierras de Córdoba, tal como se ha informado en otros sistemas montañosos del mundo (Grau, 2005; Behling \& Pillar, 2007; Coop et al., 2010, entre otros).

Los sitios sin quemar fueron similares en cuanto a su fisonomía, composición florística y 
características edáficas, en todas las exposiciones de ladera y posiciones topográficas. Sin embargo, los sitios quemados más recientemente tuvieron diferencias significativas entre sí, principalmente entre las distintas exposiciones de ladera. Este resultado estaría indicando que el dosel arbóreo enmascara variaciones mesoclimáticas relacionadas a las características topográficas, manteniendo constantes las características de la vegetación y del suelo (Belsky, 1994), aun cuando cambien la posición topográfica y la exposición de las laderas.

Es interesante destacar que la fisonomía y la composición florística tuvieron respuestas diferentes entre sitios recientemente quemados. Según la fisonomía los sitios quemados hace 4 años se diferenciaron de los sitios quemados hace 10-17 y 2 años; los primeros se caracterizaron por una alta cobertura del estrato arbustivo, mientras que los segundos presentaron una mayor cobertura del estrato herbáceo. Por otro lado, según su composición florística las tres situaciones fueron significativamente diferentes entre sí. Este desacople en la respuesta de la fisonomía y la composición florística puede deberse a que los sitios quemados hace $10-17$ y 2 años comparten su fisonomía de pastizal pero no su composición florística. En el primer caso, los sitios quemados hace 10-17 años se encuentran dominados por la gramínea en mata Festuca hieronymi, mientras que los sitios quemados hace dos años se caracterizan por la presencia de Jarava pseudoichu, gramínea en mata que crece a menor altitud que $F$. hieronymi y que es frecuente en sitios dominados por A. caven (Giorgis et al., 2011a) (Eje 2 del PCoA, Fig. 4a y 4b). Por otro lado, las diferencias florísticas entre los sitios quemados hace 2 y 4 años, se deben a que los segundos tienen una mayor proporción de especies características de los sitios sin quemar. Estas diferencias están reflejando una gran heterogeneidad espacial en la composición florística y fisonomía del Bosque Serrano. Puntualmente, parte de las diferencias pueden estar relacionadas a la una gran capacidad de rebrote reportada en las especies arbóreas del Bosque Serrano (Gurvich et al., 2005; Torres, 2012), que podrían estar explicando la recuperación de especies leñosas en los sitios quemados hace 4 años. Asimismo, los sitios quemados hace 2 años además de ser los sitios con menor tiempo transcurrido desde el último incendio, sufrieron otro fuego dos años antes, mostrando por ello una menor representación de especies características de los sitios sin quemar.

La riqueza de especies fue significativamente menor en los sitios no quemados que en las tres restantes edades post-fuego, en coincidencia con resultados reportados para numerosas regiones del mundo (DeGrandpré et al., 1993; Rees \& Juday, 2002; Verzino et al., 2005; Coop et al., 2010). Sin embargo, no se encontraron diferencias significativas de la riqueza de especies entre las tres edades post-fuego más recientes. Este último hecho nuevamente puede deberse al efecto de factores como la herbivoría y la historia de fuegos. Asimismo, los suelos de los sitios sin quemar presentaron mayores porcentajes de materia orgánica y de nitrógeno, carbono y calcio comparados con los suelos de los sitios recientemente quemados. Como ha sido reportado generalmente para ecosistemas boscosos del mundo (De Luis et al., 2003; GonzálesPérez et al., 2004) y de la provincia de Córdoba (González et al., 1999; Abril et al., 1999; Abril et al., 2005), las propiedades del suelo se ven alteradas por el fuego. Esto es aún más evidente teniendo en cuenta el gran volumen de materiales leñosos de las fisonomías boscosas, cuyos incendios pueden alcanzar altas temperaturas, produciendo drásticas modificaciones en las propiedades del suelo (Miglietta, 1994; Albanesi \& Anriquez, 2004). Debido a las grandes pendientes de esta zona y a la presencia de ganado doméstico, es muy probable que el suelo descubierto por los incendios quede sujeto a procesos erosivos y pérdida de material. Como resultado se forman suelos arenosos, con rocas sueltas y bajo contenido de materia orgánica, como fue informado en este trabajo para todos los sitios quemados más recientemente, especialmente para los sitios quemados hace 10-17 años, en posiciones topográficas altas y en sitios con bajas pendientes. Otro factor que podría estar explicando la baja concentración de materia orgánica en suelos dominados por gramíneas en mata, es la calidad de la broza aportada por la vegetación (Chapin et al., 2002). Ya que en estas comunidades la broza tiene en general bajo contenido de nitrógeno y alto contenido de fibras (suma de celulosa, hemicelulosa y lignina), que se traducen en un material difícil de descomponer y en consecuencia una menor tasa de formación de materia orgánica (Cornelissen et al., 1999; Vaieretti et al., 2005; Pérez-Harguindeguy et al., 2007). 


\section{Agradecimientos}

A los siguientes colegas por su colaboración en el trabajo de campo: D. E. Gurvich y A. Pais Bosch. A los miembros del Museo Botánico (IMBIV), particularmente a sus asistentes del Herbario: J. Ponce, P. Hick, A. Bringas y de la Biblioteca: B. Pombo. A F. Chiarini, J. Chiapella, G. Barboza, L. E. Ariza, A. A. Coccuci y P. Demaio por su ayuda con la identificación de algunas especies. A personal de Defensa Civil de Río Ceballos por la colaboración en la reconstitución de la historia de fuegos. A V. Vaieretti y a dos revisores anónimos por sus comentarios, que han contribuido a mejorar la calidad del manuscrito. Este trabajo fue financiado por SECYT, Universidad de Córdoba, y el Inter-American Institute for Global Change Research (IAI) CRNII-2005. Los autores agradecen al Consejo Nacional de Investigaciones Científicas y Técnicas (CONICET) y a la Universidad Nacional de Córdoba por las facilidades brindadas para llevar a cabo esta investigación. La primera autora es investigadora de CONICET.

\section{Bibliografía}

ABRIL, A. \& C. GONZÁLEZ. 1999. Dinámica de la fertilidad y de las poblaciones microbianas en suelos afectados por incendios en las sierras de Córdoba (Argentina). Agriscientia XVI: 63-70.

ABRIL, A., P. BARTTFELD \& E.H. BUCHER. 2005. The effect of fire and overgrazing disturbes on soil carbon balance in the Dry Chaco forest. Forest Ecol. Manag. 206: 399-405.

ALBANESI, A. \& A. ANRIQUEZ. 2003. El fuego y el suelo. In: KUNST C., BRAVO S. \& PANIGATTI J.L. (eds.), Fuego en los ecosistemas Argentinos, pp. 47-60. INTA - EEA Santiago del Estero.

ANCHORENA, J. \& A.M. CINGOLANI. 2002. Identifying habitat types in a disturbed area of the forest-steppe ecotone of Patagonia. Plant Ecol. 158: 97-112.

ARIZA, L.E. 2000. Familia Asteraceae: Tribu Heliantheae. Pródromo, de la flora fanerogámica de Argentina Central. N². Museo Botánico (IMBiV-UNC).

ARIZA, L.E. 2008. Familia Asteraceae: Tribu Inuleae (excepto Achyrocline, Gamochaeta y Gnaphalium).

Pródromo, de la flora fanerogámica de Argentina Central. N4. Museo Botánico (IMBiV-UNC).

BATLLORI, E. \& E. GUTIÉRREZ. 2008. Regional tree line dynamics in response to global change in the Pyrenees. J. Ecol. 96: 1275-1288.

BELNAP, J. \& D. ELDRIDGE. 2001. Disturbance and Recovery of Biological Soil Crusts. Chapter 27. In: J. BELNAP \& O.L. LANGE (eds), Biological soil crusts: structure, function, and management, pp 363-383. Springer-Verlag, Berlin, Germany.

BELSKY, J.A. 1994. Influences of Trees on Savanna Productivity: Tests of Shade, Nutrients, and TreeGrass Competition. Ecology 75: 922-932.

BERTRÁN DE SOLÍS, A. 1986. Estructura y composición de la comunidad de bosque serrano. In: ACOSTA, A., E. ALESSANDRIA, L. ARGÜELLO, A. BARCHUK, A.M. BERTRÁN DE SOLÍS, R. BREIMER, M. CABIDO, J. CASERNEIRO, C. ESTRABOU, F.M. GALERA, E. GÓMEZ MOLINA, J. KEEGAN, R. KOPTA, R. LUTI, N. MARTIJENA, A. PALACIOS, D. SIPOWICZ, G. VEGA, J.L. ZAMAR \& D. AVAL-SOLIS (eds.), Efecto de las actividades humanas sobre los ecosistemas montañosos y de tundra. Proyecto regional andino. Pachón-achala, pp. 97-111. UNESCO, Montevideo.

BOND, W.J. \& J.E. KEELEY. 2005. Fire as a global 'herbivore': the ecology and evolution of flammable ecosystems. Trends Ecol. Evol. 20: 387-394.

BOWMAN, D.M.J.S., J.K. BALCH, P. ARTAXO, W.J. BOND, J.M. CARLSON, et al., 2009. Fire in the earth system. Science 324: 481-484.

BREMNER, J.M. 1996. Nitrogen - total. Chapter 37. In: DL Sparks (ed.), Methods of Soil Analysis. Part 3 - Chemical Methods ASA, SSSA, CSSA, pp. 1085-122. Madison, Wisconsin.

CABIDO, M., M.L. CARRANZA, A. ACOSTA \& S. PÁEZ. 1991. Contribución al conocimiento fitosociológico del Bosque Chaqueño Serrano en la provincia de Córdoba, Argentina. Phytocoenología 19: 547-566.

CABRERA, A. 1976. Regiones fitogeográficas argentinas. Enciclopedia Argentina de Agricultura y Jardinería. 2nd ed. ACME, Buenos Aires.

CAIRNS, D.M. \& J. MOEN. 2004. Herbivory influences tree lines. J. Ecol. 92: 1019-1024.

CAPITANELLI J. 1979. Geomorfología. In: VAZQUEZ, J., R. MIATELLO \& M. ROQUE (eds.), Geografía Física de la Provincia de Córdoba, pp. 213-296. Editorial Boldt, Buenos Aires.

CAPITANIO, C. \& R. CARCAILLET. 2008. Post-fire Mediterranean vegetation dynamics and diversity: A discussion of succession models. Forest Ecol. Manag. 255: 431-439.

CARCAillet, C., A.A. Ali, O. Blarquez, A. GENRIES, B. MOURIER \& L. BREMOND. 2009. Spatial variability of fire history in subalpine forests: from natural to cultural regimes. Ecoscience 16: $1-12$. 
CARIGNANO, C.A. \& M.A. CIOCCALE. 1992. Erosion taken place in mountainous regions by effect of the forest fires. II Colombian conference of Environmental Geology. Colombia 541 pp.

CARIGNANO, C.A. 1999. Late Pleistocene to recent climate change in Córdoba Province, Argentina: Geomorphological evidence. Quatern. Int. 57/58: 117-134.

CERDA,A. \& S.H. DOERR. 2005. Influence of vegetation recovery on soil hydrology and erodibility following fire: an 11-year investigation. Int. J. Wildland Fire 14: 423-437.

CHAPIN, S. F. III, P.A. MATSON \& H. A. MONEY. 2002. Principles of Terrestrial Ecosystem Ecology. Springer-Verlag. New York.

CHAZDON, R.L. 2003. Tropical forest recovery: legacies of human impact and natural disturbances. Perspect. Plant Ecol. 6: 51-71.

CIERJACKS, A., K. WESCHE \& I. HENSEN. 2007. Potential lateral expansion of Polylepis forest fragments in central Ecuador. Forest Ecol. Manag. 242: 477-486.

CINGOLANI, A.M., D. RENISON, P.A. TECCO, D.E. GURVICH \& M. CABIDO. 2008. Predicting cover types in a mountain range with long evolutionary grazing history: a GIS approach. J. Biogeogr. 35: 538-551.

CIOCCALE, M. 1999. Geomorfología hidrica de un sector de las Sierras de Córdoba. Tesis doctoral en Geología, Universidad Nacional de Córdoba.

COLLINS, B.M, M. KELLY, J.W. VAN WAGTENDONK \& S.L. STEPHENS. 2007. Spatial patterns of large natural fires in Sierra Nevada wilderness areas. Landscape Ecol. 22:545-557.

COOP, J.D., R.T. MASSATTI \& A.W. SCHOETTLE. 2010. Subalpine vegetation pattern three decades after stand-replacing fire: effects of landscape context and topography on plant community composition, tree regeneration, and diversity. J. Veg. Sci. 21: 472-487.

CORNELISSEN, J.H.C., N. PÉREZ-HARGUINDEGUY, S. DÍAZ, J. P.GRIME, B. MARZANO, M. CABIDO, F. VENDRAMINI \& B. CERABOLINI. 1999. Leaf structure and defence control litter decomposition rate across species and life forms in regional floras on two continents. New Phytol. 143: 191-200.

DAY, P.R. 1986. Particle fractionation and particle size analysis. In: Klute, CA, ed. Methods of soil analysis. Part 1. Physical and mineralogical methods, pp.545567. 2nd ed. Madison, American Society Agronomy.

DE LUIS, M., J.C. GONZÁLEZ-HIDALGO \& J. RAVENTÓS. 2003. Effects of fire and torrential rainfall on erosion in a Mediterranean gorse community. Land Degrad. Dev. 14: 203-213.

DEGRANDPRÉ, L., D. GAGNON \& Y. BERGERON.
1993. Changes in the understory of Canadian southern boreal forest after fire. J. Veg. Sci. 4: 803-810.

DEMAIO, P. H., M.H. J. BARFUSS, R. KIESLING, W. TILL, \& J.O. CHIAPELLA. 2011. Molecular phylogeny of Gymnocalycium (cactaceae):assessment of alternative infrageneric systems, a new subgenus, and trends in the evolution of the genus. Amer. J. Bot. 98: 1841-1854.

DULLINGER, S., T. DIRNBÖCK \& G. GRABHERR. 2004. Modelling climate change-driven tree line shifts: relative effects of temperature increase, dispersal and invasibility. J. Ecol. 92: 241-252

EUGENE, M. \& F. LLORET. 2004. Fire recurrence effects on the structure and composition of Mediterranean Pinus halepensis communities in Catalonia (northeast Iberian Peninsula). Ecoscience 11: 455-462.

FULÉ, P.Z. \& D.C. LAUGHLIN. 2007. Wildland fire effects on forest structure over an altitudinal gradient, Grand Canyon National Park, USA. J. Appl. Ecol. 44: 136-146.

FUNES, G. \& M. CABIDO. 1995. Variabilidad local y regional de la vegetación rupícola de las Sierras Grandes de Córdoba, Argentina. Kurtziana 24: $173-$ 188.

GAVIER, G.I. \& E.H. BUCHER. 2004. Deforestación de las Sierras Chicas de Córdoba (Argentina) en el período 1970-1997. Academia Nacional de Ciencias. Miscelánea $\mathrm{N}^{\mathrm{0}} 101$.

GAVIER-PIZARRO, G.I., T. KUEMMERLE, L.E. HOYOS, S.I. STEWART, C.D. HUEBNER, N.S. KEULER, V.C. RADELOFF. 2012. Monitoring the invasion of an exotic tree (Ligustrum lucidum) from 1983 to 2006 with 1 Landsat TM/ETM+ satellite data and support vector machines in Córdoba, Argentina. Remote sens environ. doi:10.1016/j. rse.2011.09.023.

GENRIES, A, S.D. MULLER, L. MERCIER, L. BIRCKER \& C. CARCAILlET. 2009. Fires Control Spatial Variability of Subalpine Vegetation Dynamics During the Holocene in the Maurienne Valley (French Alps). Ecoscience 16: 13-22.

GIBSON, D.J. \& L.C. HULBERT. 1987. Effects of fire, topography and year-to-year climatic variation on species composition in tallgrass prairie. Plant Ecol. 72: 175-185.

GIORGIS, M.A., A.M. CINGOLANI, F. CHIARINI, J. CHIAPELLA, G. BARBOZA, ARIZA ESPINAR, L., MORERO, R., GURVICH, D.E., TECCO, P.A., SUBILIS, R., \& M. CABIDO. 2011b. Composición florística del Bosque Chaqueño Serrano de la provincia de Córdoba, Argentina. Kurtziana 36: 9-43.

GIORGIS, M.A., A.M. CINGOLANI, I. TEICH \& R. RENISON. 2010. Do Polylepis australis trees tolerate herbivory? Seasonal patterns of biomass 
production and its consumption by livestock. Plant Ecol. 207: 307-319.

GIORGIS, M.A., M. CABIDO \& A.M. CINGOLANI. 2011a. Caracterización florística y estructural del Bosque Chaqueño Serrano. Editorial Académica Española (EAE), Berlín. ISBN 978-3-8443-4123-2.

GIORGIS, M.A., P. TECCO, A.M. CINGOLANI, P. MARCORA, D. RENISON \& V. PAIARO. 2011c. Factors associated with woody alien species distribution in a newly invaded mountain system of central Argentina. Biol. Invasion. 13: 1423-1434.

GONZÁLES-PÉREZ, J.A., F.J. GONZÁLES-VILA, G. ALMENDROS \& H. KNICKER. 2004. The effect of fire on soil organic matter-a review. Environ. Int. 30: 855-870.

GONZÁlez, C., A. ABRIL \& M. ACOSTA. 1999. Efecto del fuego sobre las comunidades microbianas y la fertilidad edáfica en el Chaco Occidental. Ecología Austral 9: 3-10.

GORDILLO, C.E. 1979. Observaciones sobre la petrología de las rocas cordieríticas de las Sierras de Córdoba. Bol. Acad. Nac. Cs. 53: 3-44.

GRAU, H.R. \& R. ARAGÓN. 2000. Árboles Invasores de la Sierra de San Javier, Tucumán Argentina. In: GRAU, H.R. \& R. ARAGÓN (eds.), Ecología de árboles exóticas en las yungas Argentinas. pp 3-21. LIEY, Tucumán.

GRAU, H.R. 2003. Ecología del fuego en los ecosistemas montanos superiores del subtrópico. In: KUNST, C., BRAVO, S. \& J.L. PANIGATTI (eds.), Fuego en los ecosistemas Argentinos. pp. 181-188.INTA, EEA. Santiago del Estero.

GRAU, H.R. 2005. Dinámica de bosques en el gradiente altitudinal de las Yungas Argentinas. In: ARTURI, M. FRANGI, J. \& J. GOYA (eds.). Ecología y Manejo de los Bosques Argentinos, pp. 181-188. Universidad Nacional de La Plata.

GURVICH, D.E., L. ENRICO \& A.M. CINGOLANI. 2005. Linking plant functional traits with postfire sprouting vigour in woody species in central Argentina. Austral Ecology 30: 789-796.

HARSCH, M.A., P.E. HULME, M.S. MCGLONE \& R.P. DUNCAN. 2009. Are treelines advancing? A global meta-analysis of treeline response to climate warming. Ecol. Lett. 12: 1040-1049.

HOYOS, L.E., G.I. GAVIER-PIZARRO, T. KUEMMERLE, E.H. BUCHER, V.C. RADELOFF \& P.A. TECCO. 2010. Invasion of glossy privet (Ligustrum lucidum) and native forest loss in the Sierras Chicas of Córdoba, Argentina. Biol. Invasions. 12: 3261-3275.

JOHANSEN, J. R. 2003. Impacts of fire on biological soil crusts. In: BELNAP, J. \& O.L. LANGE (eds.), Biological soil crusts: structure, function, and management. Ecological Studies 150, pp. 385-397. Springer-Verlag, Berlin.

KITZBERGER, T., P.M. BROWN, E.K. HEYERDAHL, T.W. SWETNAM \& T.T. VEBLEN. 2007. Contingent Pacific-Atlantic Ocean influence on multicentury wildfire synchrony over western North America. Proc. Natl. Acad. Sci. 104: 543-8.

KÖRNER, Ch. \& J. PAULSEN. 2004. A world-wide study of high altitude treeline temperatures. $J$. Biogeogr. 31: 713-732.

KRAMER, K., T.A. GROEN \& S.E. VAN WIEREN. 2003. The interacting effects of ungulates and fire on forest dynamics: an analysis using the model FORSPACE. Forest Ecol. Manag. 181: 205-222.

KRAMER, M.G., A.J. HANSEN, M.L. TAPER \& E.J. KISSINGER. 2001. Abiotic controls on long-term windthrow disturbance and temperate rain forest dynamics in southeast Alaska. Ecology 82: 27492768.

KULAKOWSKI, D. \& T.T. VEBLEN. 2002. Influences of Fire History and Topography on the Pattern of a Severe Wind Blowdown in a Colorado Subalpine Forest. J. Ecology. 90: 806-819.

KUNST, C. \& S. BRAVO. 2003. Fuego, calor y temperatura. In: KUNST C., BRAVO S. \& J.L. PANIGATTI (eds.), Fuego en los ecosistemas Argentinos, pp. 47-60. INTA, EEA Santiago del Estero.

KUO, S. 1996. Phosphorus. Chapter 32. In DL Sparks (ed.), Methods of Soil Analysis. Part 3-Chemical Methods ASA, SSSA, CSSA, pp. 869-920. Madison, Wisconsin.

KURTZ, F. 1904. Flora de Córdoba. In: RÍO, M. E. \& L. ACHÁVAL (eds.), Geografía de la Provincia de Córdoba. Vol. 1, pp. 270-343. Compañía Sudamericana de Billetes de Banco, Buenos. Aires.

LAVEEA, H., P. KUTIEL, M. SEGEVA \& Y. BENYAMINIA. 1995. Effect of surface roughness on runoff and erosion in a mediterranean ecosystem: the role of fire. Geomorphology 11: 227-234.

LECOMTE, N., M. SIMARD, N. FENTON \& Y. BERGERON. 2006. Fire Severity and Long-term Ecosystem Biomass Dynamics in Coniferous Boreal Forests of Eastern Canada. Ecosystems 9: 1215-1230.

LUTI, R., M.A. BERTRÁN DE SOLÍS, M.F. GALERA, N. MÜLLER DE FERREIRA, M. BERZAL, M. NORES, M.A. HERRERA \& J.C. BARRERA. 1979. Vegetación. In: J. VÁZQUEZ, R. MIATELLO \& M. ROQUE (eds.), Geografía Física de la provincia de Córdoba, pp. 297-368. Ed. Boldt, Buenos Aires.

MARCORA, P., D. RENISON, A. I. PAÍS-BOSCH, M. R. CABIDO \& P. A. TECCO. 2013. The effect of altitude and grazing on seedling establishment of woody species in central Argentina. Forest Ecol. Manag. 291: 300-307. 
MARCORA, P., I. HENSEN, D. RENISON, P. SELTMANN \& K. WESCHE. 2008. The performance of Polylepis australis trees along their entire altitudinal range: implications of climate change for their conservation. Divers. Distrib. 14: 630-636.

MARI, N. 2006. Estudio de la ocurrencia de incendios en el Valle de Punilla, Córdoba. Tesis de grado. Universidad de Ciencias empresariales y Sociales.

MARTIN, P.H., R.E. SHERMAN \& T.J. FAHEY. 2007. Tropical montane forest ecotones: climate gradients, natural disturbance, and vegetation zonation in the Cordillera Central, Dominican Republic. J. Biogeogr. 34: 1792-1806.

MIGLIETTA, S. 1994. Patrón de ocurrencia de fuegos y su efecto sobre la vegetación en el bosque Serrano de Córdoba. Tesis de Maestría, Facultad de Ciencias Exactas, Físicas y Naturales, Universidad Nacional de Córdoba, Córdoba, Argentina.

MONTAÑA, C. \& E. EZCURRA. 1982. Aplicación de algunos métodos de clasificación numérica al estudio de la vegetación de la Quebrada de Vaquerías (Valle Hermoso, Córdoba). Ecología 7: 55-66.

MONTAÑA, C. 1975. Observaciones sobre la vegetación de la Quebrada de Vaquerías, Valle Hermoso, Córdoba. Facultad de Ciencias Exactas, Físicas y Naturales. Universidad Nacional de Córdoba. (Trabajo final de Seminario). Inédito.

MOREIRA, A.G. 2000. Effects of Fire Protection on Savanna Structure in Central Brazil. J. Biogeogr. 27: 1021-1029.

MULVANEY, R.L. 1996. Nitrogen - inorganic forms. In: D. L. SPARKS (ed.), Methods of Soil Analysis. Part 3 - Chemical Methods Chapter 38. ASA, SSSA, CSSA, pp. 1123-84. Madison, Wisconsin.

NELSON, D.W. \& L.E. SOMMERS. 1996. Total carbon, organic carbon, and organic matter. In: D. L. SPARKS (ed.), Methods of Soil Analysis. Part 3 Chemical Methods, Chapter 34, pp. 961-1010. ASA, SSSA, CSSA, Madison, Wisconsin.

PAGE, C.A. 2005. El Río de los Ceballos historia de un pueblo 1583-1983. BR Copias.

PAIS BOSCH, A.I., P.A. TECCO, G. FUNES \& M. CABIDO. En prensa. Efecto del la temperatura en la regeneración de especies leñosas del Chaco Serrano e implicancias en la distribución actual y potencial de bosques. Bol. Soc. Arg. Bot.

PÉREZ HARGUINDEGUY, N., S. DÍAZ, F. VENDRAMINI, D. E. GURVICH, A. M. CINGOLANI, M. A. GIORGIS \& M. CABIDO. 2007. Direct and indirect effects of climate on decomposition in native ecosystems from central Argentina. Austral Ecol. 32: 749-757.

PILLAR, V.D. \& L. ORLÓCI. 1996. On randomization testing in vegetation science: multifactor comparisons of relevé groups. J. Veg. Sci. 7: 585-92.

PILLAR, V.D. 2004. MULTIV - Multivariate Exploratory

Analysis, Randomization Testing and Bootstrap Resampling; User's Guide v. 2.3. Departamento de Ecologia, UFRGS, Porto Alegre. Available from URL: http://ecoqua.ecologia.ufrgs.br

PODANI, J. 1999. Extending Gower's general coefficient of similarity to ordinal characters. Taxon 48: 331340 .

PODANI, J. 1994. Multivariate data analysis in ecology and systematics: a methodological guide to the SYNTAX 5.0 package. SPB Academic Publishing, The Hague, Netherlands.

RAPELA, C. 1982. Aspectos geoquímicas y petrológicos del batolito de Achala. Provincia de Córdoba. Rev. Asoc. Geol. Arg. 37: 313-330.

REES, D.C. \& G.P. JUDAY. 2002. Plant species diversity on logged versus burned sites in central Alaska. Forest Ecol. Manag. 155: 291-302.

REICH, P.B., D.W. PETERSON, D.A. WEDIN \& K. WRAGE. 2001. Fire and vegetation effects on productivity and nitrogen cycling across a forestgrassland continuum. Ecology 82: 1703-1719.

REINHARDT, L., D. JEROLMACK, B.J. CARDINALE, V. VANACKER \& J. WRIGHT. 2010. Dynamic interactions of life and its landscape: feedbacks at the interface of geomorphology and ecology. Earth Surf. Processes. 35: 78-101.

RENISON, D., A.M. CINGOLANI \& R. SUAREZ. 2002. Efectos del fuego sobre un bosquecillo de Polylepis australis (Rosaceae) en las montañas de Córdoba, Argentina. Rev. Chil. Hist. Nat. 75: 719-727.

ROLLINS, M.G., P. MORGAN \& T. SWETNAM. 2002. Landscape-scale controls over 20th century fire occurrence in two large Rocky Mountain (USA) wilderness areas. Landscape Ecol. 17: 539-557

SAYAGO, M. 1969. Estudio fitogeográfico del norte de Córdoba. Boletín de la Academia Nacional de Ciencias de Córdoba 46: 123-427.

SHERMAN, R.E., P.H. MARTIN, T.J. FAHEY \& S.D. DEGLORIA. 2008. Fire and Vegetation Dynamics in High-elevation Neotropical Montane Forests of the Dominican Republic. Ambio 37: 7-8.

SIBOLD, J.S., T.T. VEBLEN \& M.E. GONZÁLEZ. 2006. Spatial and temporal variation in historic fire regimes in subalpine forests across the Colorado Front Range in Rocky Mountain National Park, Colorado, USA. J. Biogeogr. 32: 631-647.

SPEED, J.D.M., G. AUSTRHEIM, A.J. HESTER \& A. MYSTERUD. 2010.Experimental evidence for herbivore limitation of the treeline. Ecology 91: 3414-3420.

SUMNER, M.E. \& W.P. MILLER. 1996. Cation exchange capacity and exchange coefficients. In: D. L. SPARKS (ed.), Methods of Soil Analysis. Part 3 - 
Chemical Methods, Chapter 40. ASA, SSSA, CSSA, pp. 1201-30. Madison, Wisconsin.

SWANSON, F.J., T.K. KRATZ, N. CAINE \& R.G. WOODMANSEE. 1988. Landform effects on Ecosystem Patterns and Processes. BioScience 38: 92-98.

TEICH, I., A.M. CINGOLANI, D. RENISON, I. HENSEN \& M.A. GIORGIS. 2005. Do domestic herbivores retard Polylepis australis woodland recovery in the mountains of Córdoba, Argentina? Forest Ecol. Manag. 219: 229-241.

THOMAS, G.W. 1996. Soil pH and soil acidity. In: D. L. SPARKS (ed.), Methods of Soil Analysis. Part 3 Chemical Methods, Chapter 16, pp. 475-90. ASA, SSSA, CSSA. Madison, Wisconsin.

TORRES, R.C. 2012. Efectos de la degradación del bosque serrano cordobés sobre la capacidad de regeneración natural y asistida de tres especies arbóreas de estadios maduros. Tesis doctoral. Universidad Nacional de Córdoba.

TURNER, M.G. \& W.H. ROMME. 1994. Landscape dynamics in crown fire ecosystems. Landscape Ecol. 9: 59-77.

VAIERETTI, M. V., N. PÉREZ HARGUINDEGUY, D. E. GURVICH, A. M. CINGOLANI \& M. CABIDO. 2005. Decomposition dynamic and physico-chemical of abundant species in a montane woodland central Argentina. Plant Soil 278: 223-234.
VAN DER MAAREL, E. 1979. Transformation of coverabundance values in phytosociology and its effects on community similarity. Plant Ecol. 39: 97-114.

VERZINO, G., J. JOSEAU, M. DORADO, E. GELLERT, S. RODRÍGUEZ REARTES \& R. NÓBILE. 2005. Impacto de los incendios sobre la diversidad vegetal, Sierras de Córdoba, Argentina. Ecología Aplicada 4: 25-34.

VILÁ-CABRERA, A., S. SAURA-MAS \& F. LLORET. 2008. Effects of fire frequency on species composition in a Mediterranean shrubland. Ecoscience 15: 519528.

VILLALBA, R., M.H. MASIOKAS, T. KITZBERGER \& J. BONINSEGNA. 2005. Biogeographical consequences of recent climate changes in the southern Andes of Argentina. In: HUBER, U., REASONER, M. \& H. BUGMANN (eds.), Global Change and Mountain Regions: A State of Knowledge Overview, Advances in Global Change Research, Vol. 23, pp. 157-168. Springer, Dordrecht.

ZULOAGA, F.O., O. MORRONE \& M.J. BELGRANO. 2008. Catálogo de Plantas Vasculares del Cono Sur. Monogr. Missouri Bot. Garden (USA).

Recibido el 19 de septiembre de 2012, aceptado el 1 de abril de 2013. 
